\title{
Elastic wave-equation-based reflection kernel analysis and traveltime inversion using wave mode decomposition
}

\author{
Tengfei Wang, ${ }^{1}$ Jiubing Cheng, ${ }^{1}$ Qiang Guo ${ }^{2}$ and Chenlong Wang ${ }^{1}$ \\ ${ }^{1}$ State Key Laboratory of Marine Geology, Tongji University, Shanghai 200092, China. E-mail: cjb1206@tongji.edu.cn \\ ${ }^{2}$ Department of Physical Science and Engineering, King Abdullah University of Science and Technology, Thuwal 23955-6900, Saudi Arabia
}

Accepted 2018 July 17. Received 2018 July 1; in original form 2017 September 06

\begin{abstract}
S U M M A R Y
Elastic reflection waveform inversion (ERWI) utilizes reflections to update the low and intermediate wavenumbers in the deeper part of elastic models and can provide good initial models for elastic full waveform inversion (EFWI). Although ERWI aims to mitigate the nonlinearity of inversion when starting from a poor initial model, it suffers from the cycle-skipping problem due to the objective function of waveform fitting. Building initial $P$ - and $S$-wave velocity models for EFWI through elastic wave-equation reflection traveltime inversion (ERTI) would be effective and robust since traveltime information relates to the background model more linearly. However, the current implementations of acoustic traveltime inversion is not straightforward in elastic media due to the existence of $S$-wavefields. Wave mode decomposition, both on the recording surface and in the extrapolated wavefields, is important for ERTI. First, for seismic data with $P$-wave sources, the $P / S$ separation of multicomponent seismograms isolates the $P P$ and $P S$ reflection events and thus make it possible to extract the event-to-event time-shifts of these isolated reflections through dynamic image warping (DIW). Then, we can use the traveltime residuals of $P P$ and $P S$ reflections to build the objective function for ERTI. Second, based on the investigation of the complicated reflection kernels in an elastic medium, we demonstrate the necessity of wave mode decomposition applied on the extrapolated elastic wavefields, to suppress the artefacts induced by the undesirable cross-correlations of the components in forward and back-propagated wavefields. Therefore, the decomposition of surface recording data and extrapolated wavefields guarantees the dominate contribution of the traveltime is included during the ERTI. Accordingly, we propose a two-stage method to first build the $P$-wave background velocity using the separated $P P$ reflections and then build the $S$-wave background velocity using the separated $P S$ reflections based on the well-recovered $P$-wave velocity model. A numerical example of the Sigsbee2A model shows the effectiveness of the proposed ERTI approach.
\end{abstract}

Key words: Inverse theory; Waveform inversion; Seismic tomography; Elasticity and anelasticity.

\section{INTRODUCTION}

With the emergence of long-offset wide-azimuth acquisitions and broad-band sources, full waveform inversion (FWI) has been recognized as an efficient tool for constructing velocity models and for quantitative seismic imaging Virieux \& Operto (2009). Although FWI, which primarily focuses on $P$-wave velocity inversion, has been widely studied in past decades Tarantola (1984); Pratt et al. (1998); Shipp \& Singh (2002). Researchers have recently given more attention to waveform inversion under the elastic assumption, which is referred to as elastic full waveform inversion (EFWI; Tarantola 1986). Waveform inversion provides high-resolution model estimation of the elastic properties, but it suffers from the cycle skipping easily because of its insensitivity to the low and intermediate wavenumber components of the model when the acquisition illumination is poor and/or good initial models are unavailable Sears et al. (2008); Brossier et al. (2009). Moreover, multiparameter tradeoff effects and more complicated elastic wave phenomena increase the difficulties with EFWI. Appropriate parametrization, more preconditioning and hierarchical strategies should be considered during EFWI to address the nonlinearity and parameter trade-offs Sears et al. (2008); Operto et al. (2013); Prieux et al. (2013); Wang et al. (2015b); Oh \& Alkhalifah (2016).

For classic FWI, long-offset data corresponding to diving waves are important to build the long-to-intermediate wavelengths of the model. However, the penetration depths of diving waves are far 
from sufficient to reach the target in the deeper part, even when using the wide-aperture surveys. In addition, the low signal-to-noise ratio at the far offset is also a limit for FWI relying on diving waves. Therefore, researchers have attempted to utilize the reflections to help to build a macro-model containing low-to-intermediate wavenumbers in the deep part Stork (1992); Chavent et al. (1994); Clement et al. (2001); Symes (2008b); Xu et al. (2012). This process can be implemented in the image domain or the data domain. Indeed, image-domain ray-based tomography Stork (1992); Woodward et al. (2008); Jones (2010) is a workhorse in the standard workflow to obtain the background velocity by flattening the common image gathers. However, when the lateral velocity variation is strong, the ray-based method fails to present the wave propagation underground. Great efforts have been made to develop wave-equation-based reflection inversion that employs waveform or traveltime information to overcome the limits of ray theory Plessix et al. (1999); Xu et al. (2012); Ma \& Hale (2013); Chi et al. (2015); Wang et al. (2015a); Wu \& Alkhalifah (2015); Zhou et al. (2015).

Alternatively, the misfit function of reflection inversion can be built in the image domain in the manner of wave-equation migration velocity analysis (WEMVA), which aims to annihilate the energy at non-zero subsurface offsets in the image gathers Symes (2008a); Almomin \& Biondi (2012); Sun \& Symes (2012); Biondi \& Almomin (2013). Recently, Raknes \& Weibull (2016) developed an image-domain method to recover the $P$-wave velocity $\left(V_{\mathrm{p}}\right)$ in $3-\mathrm{D}$ elastic media. Wang et al. (2017a) exploited the extended $P S$ image in WEMVA to update the $S$-wave velocity $\left(V_{\mathrm{s}}\right)$ with the help of elastic wave mode decomposition. However, the extended-domain methods are limited due to their prohibitive computational cost, especially in 3-D cases. In the data domain, inspired by the pioneering work of reflection inversion Chavent et al. (1994); Plessix et al. (1999); Clement et al. (2001), Xu et al. (2012) proposed reflection waveform inversion (RWI) to reduce the nonlinearity in FWI by reconstructing the long-wavelength components of the model using the reflections predicted through a migration/demigration process. Recently, Zhou et al. (2015) proposed a joint FWI method that combines RWI and FWI to utilize both diving and reflected waves. Wu \& Alkhalifah (2015) developed an RWI scheme to simultaneously update the background velocity and the perturbation in acoustic media. This approach was recently extended to elastic media Guo \& Alkhalifah (2017), referred to as elastic reflection waveform inversion (ERWI). Theoretically, RWI requires the high-wavenumber model perturbation to generate the reflections which can match the amplitude of the observed data. And this high-wavenumber perturbation should be updated with the least-squares migration as long as the background velocity changes.

Compared with waveform information, traveltime is more sensitive and linearly related to the low-wavenumber components of the model. Therefore, traveltime inversion will be more robust and helpful to build good initial models for conventional FWI Wang et al. (2014). Ma \& Hale (2013) introduced a wave-equation reflected traveltime inversion method based on dynamic image warping (DIW) to build the low-wavenumber portion of the model. Chi et al. (2015) and Wang et al. (2015a) employed a correlation-based method to extract temporal and spatial lag to implement reflection inversion. Elastic reflections carry the background information of $V_{\mathrm{p}}$ and $V_{\mathrm{s}}$, which can help to build good initial velocity models for EFWI. Unfortunately, the current traveltime inversion method in acoustic media is hard to straightforwardly implement in elastic case. Even using the reflection tomography, we need the a priori information of important horizons and employ the particular $P$ or
$S$ events to constrain the inversion Broto et al. (2003); Du et al. (2012); Yang et al. (2015).

In elastic media, the traveltime shifts of a particular wave mode are difficult to extract due to the complicated wave phenomena, such as mode conversion. Therefore, the estimated time-shifts would be inaccurate or misleading when the original multicomponent seismograms are used directly. In addition, since the multiparameter trade-offs increase the nonlinearity of inversion, more hierarchical strategies should be considered to address this problem. Wang \& Cheng (2017) proposed preconditioning of the EFWI gradients through wave mode decomposition to mitigate the parameter tradeoffs and explained that this preconditioning approximately employs the off-diagonal Hessian blocks when recovering $V_{\mathrm{s}}$. As a natural way to obtain the separated data subsets, wave mode decomposition is expected to have the potential to precondition elastic waveequation reflection traveltime inversion (ERTI) with a more flexible hierarchical strategy.

In this paper, we exploit the traveltime misfits of the $P P$ and $P S$ reflections to implement an ERTI approach with the aid of wave mode decomposition and DIW. First, the elastic reflection kernels are calculated and decomposed according to the wave mode to obtain insights and suppress the artefacts in the gradient calculation. Then, $P / S$ separation is applied to the observed and predicted seismograms to extract the isolated event-to-event traveltime residuals of the $P P$ or $P S$ reflections via DIW. These processes lead to a twostage ERTI approach, in which we first invert the $P P$ reflections to recover the background $V_{\mathrm{p}}$ model and then invert the $P S$ reflections to recover the background $V_{\mathrm{s}}$ model. Given that the reflectors in the $P S$ image should be at the same depths as those in the $P P$ image, we take the well-positioned $P P$ image as the parameter perturbation to generate $P S$ reflections in the second stage. Moreover, we precondition the gradient with respect to $V_{\mathrm{s}}$ through mode decomposition to suppress the artefacts. A numerical example of the Sigsbee2A model is presented to illustrate the robustness and validity of our ERTI method.

\section{THEORY OF ERTI}

The subsurface of the Earth can be considered as elastic media under the elastodynamic assumption. Seismic wave propagation in such media is governed by the wave equation

$\rho \frac{\partial u_{i}^{2}}{\partial t^{2}}-\frac{\partial}{\partial x_{j}}\left[c_{i j k l} \frac{\partial u_{k}}{\partial x_{l}}\right]=f_{i}$,

where $u_{i}$ and $f_{i}$ are the $i$ th components of the particle displacement vector and the body force, respectively; $\rho$ is the density; and $c_{i j k l}$ is the component of the stiffness tensor. All indices change from 1 to 3, and Einstein's summation convention over repeated indices is implied.

Assume that there is a perturbation $c_{i j k l}^{1}$ in the background elastic media $c_{i j k l}^{0}$, the background wavefields $u_{i}$ and perturbed wavefields $\hat{u}_{i}$ satisfy

$\rho \frac{\partial u_{i}^{2}}{\partial t^{2}}-\frac{\partial}{\partial x_{j}}\left[c_{i j k l}^{0} \frac{\partial u_{k}}{\partial x_{l}}\right]=f_{i}$

and

$\rho \frac{\partial \hat{u}_{i}^{2}}{\partial t^{2}}-\frac{\partial}{\partial x_{j}}\left[c_{i j k l}^{0} \frac{\partial \hat{u}_{k}}{\partial x_{l}}\right]=\frac{\partial}{\partial x_{j}}\left[c_{i j k l}^{1} \frac{\partial u_{k}}{\partial x_{l}}\right]$.

In the sense of first-order Born scattering, $\hat{u}_{i}$ can be taken as the demigrated reflection data using the image perturbation $c_{i j k l}^{1}$ obtained via reverse time migration (RTM) or other imaging methods. 


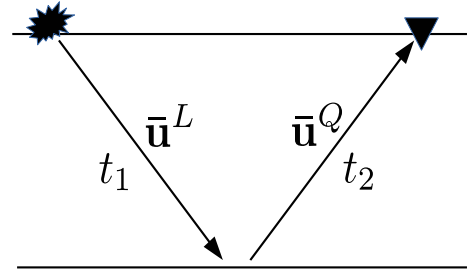

(a)

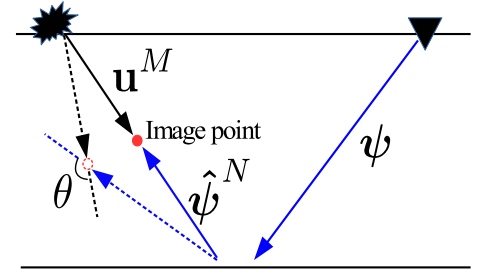

(b)

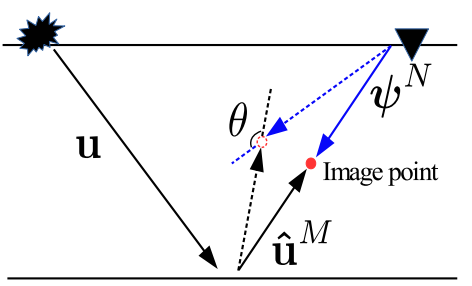

(c)

Figure 1. Schematic illustration of the calculation of the reflection kernels: (a) the physical realization of an $L$ mode converts to a $Q$ mode: $t_{1}$ and $t_{2}$ are traveltime of the incident and reflected part, respectively. (b) Source side cross-correlation satisfying $t\left(\mathbf{u}^{M}\right)+t\left(\hat{\boldsymbol{\psi}}^{N}\right)+t(\boldsymbol{\psi})=t_{1}+t_{2}$. (c) Receiver side cross-correlation satisfying $t(\mathbf{u})+t\left(\hat{\mathbf{u}}^{M}\right)+t\left(\boldsymbol{\psi}^{N}\right)=t_{1}+t_{2}$. Note, the black lines denote forward wave propagation while the blue ones denote backward wave propagation. $\overline{\mathbf{u}}$ represents the physical realization and $\mathbf{u}$ represents the numerically reconstructed wavefields in the subsurface. For a certain physical realization (a), the corresponding migration or inversion process may generate all kinds of wave modes during the wavefield extrapolation (b or c). The two cross-correlations can be taken as pre-stack migration above the interface with the receiver or source moving to their mirror locations below the interface, given that the forward and adjoint wavefields meet at the image point with a 'scattering' angle $\theta$. The common-mode cross-correlations provide zero-wavenumber (transmission) components only when $\theta=180^{\circ}$ and $M=N=L$ at source side (or $M=N=Q$ at receiver side). The low-wavenumber (transmission) components decrease if $\theta$ decreases within the first Fresnel zone. Other responses with smaller 'scattering' angles lead to relatively high-wavenumber artefacts to the reflection kernels.

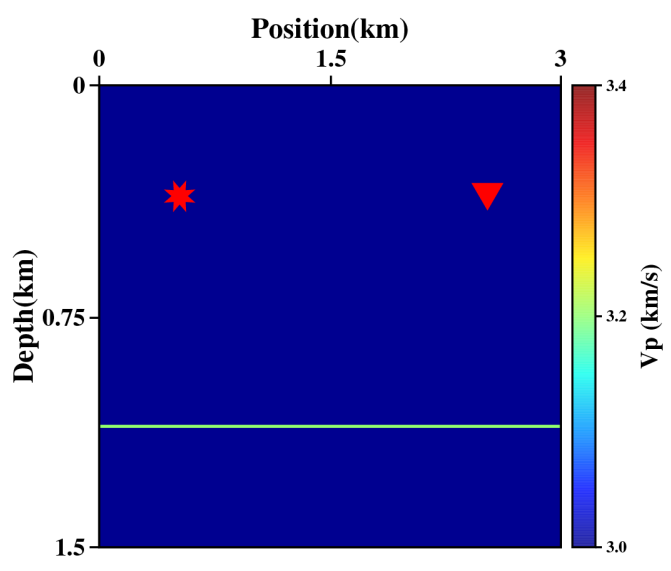

(a)

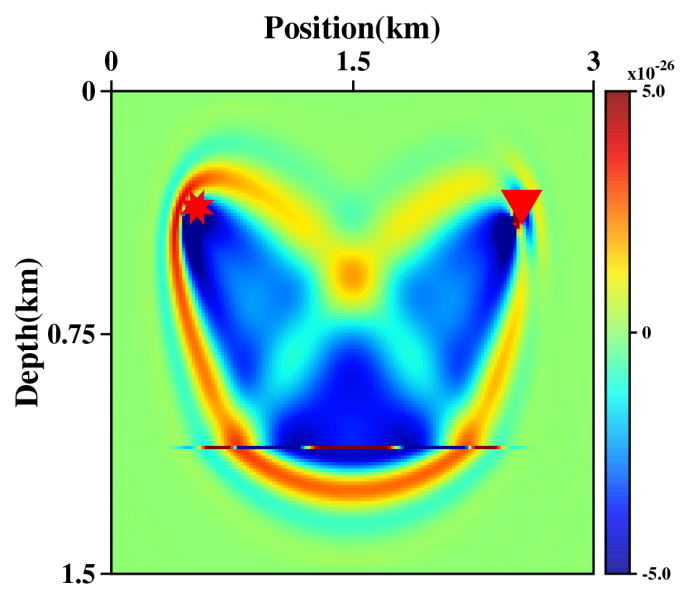

(c)

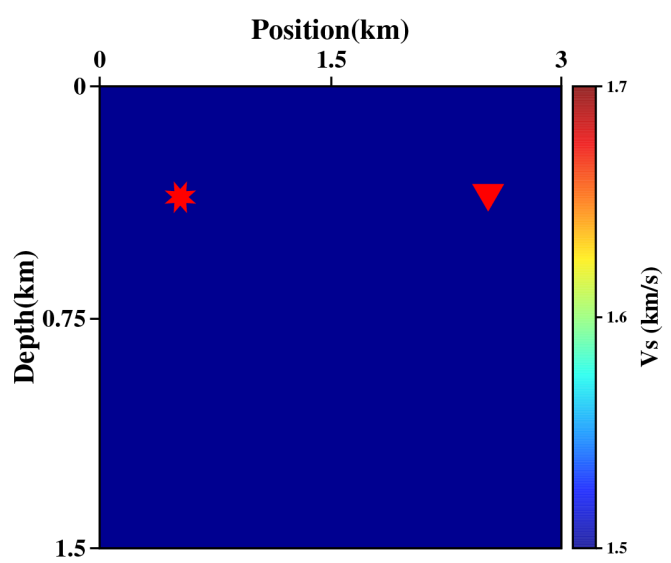

(b)

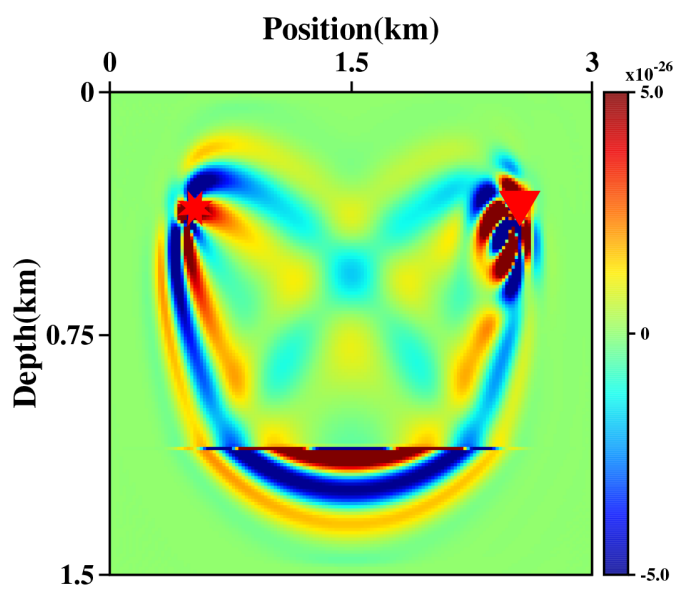

(d)

Figure 2. Reflection kernels for a horizontal interface due to the perturbation of $P$-wave velocity at the depth of $1.1 \mathrm{~km}$ : (a) $V_{\mathrm{p}}$, (b) $V_{\mathrm{s}}$, (c) $K_{V_{\mathrm{p}}}$ and (d) $K_{V_{\mathrm{s}}}$. 


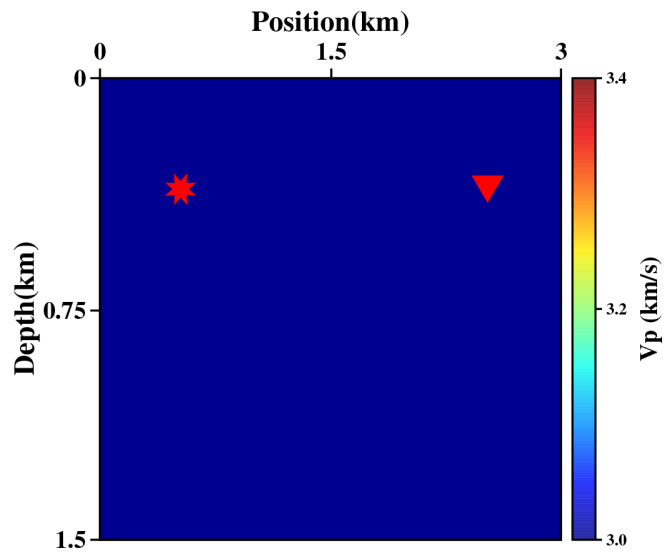

(a)

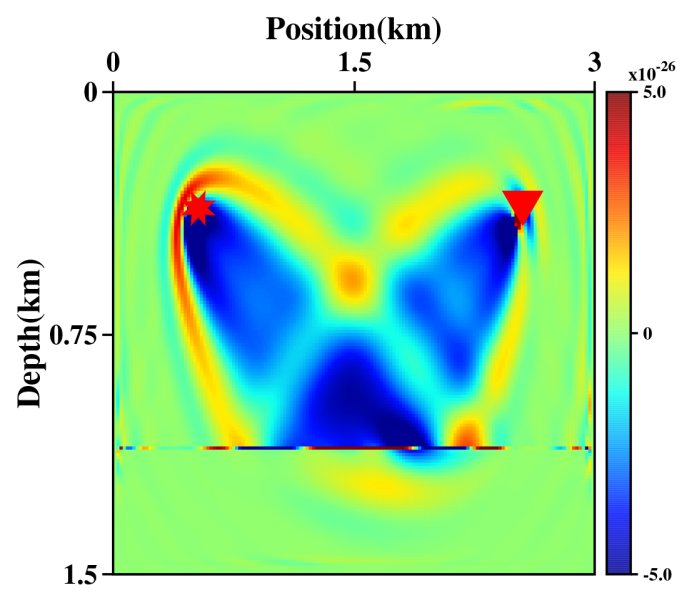

(c)

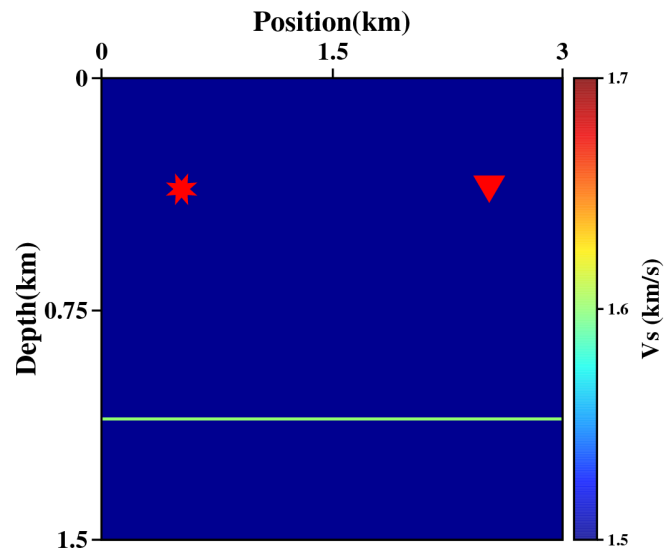

(b)

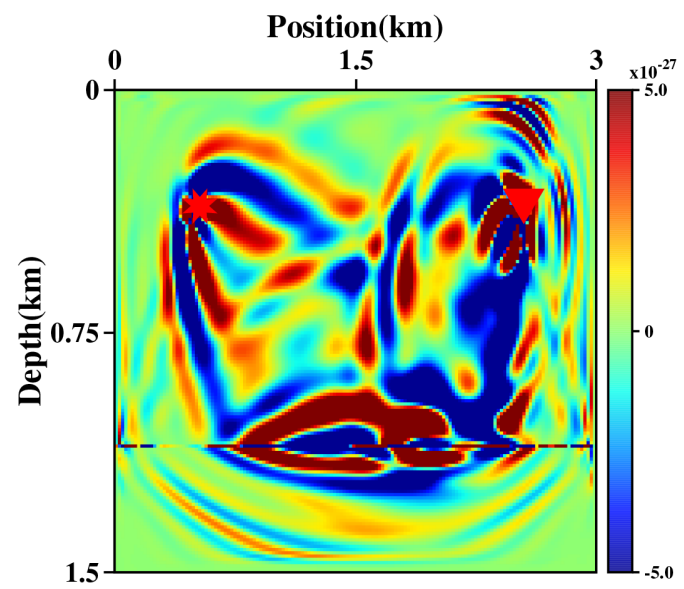

(d)

Figure 3. Reflection kernels for a horizontal interface due to the perturbation of $S$-wave velocity at the depth of $1.1 \mathrm{~km}$ : (a) $V_{\mathrm{p}}$, (b) $V_{\mathrm{s}}$, (c) $K_{V_{\mathrm{p}}}$ and (d) $K_{V_{\mathrm{s}}}$.

Due to the waveform fitting objective function in RWI, a leastsquares or true amplitude migration is required for the perturbation of stiffness tensor to accurately predict the amplitude of reflection data. In ERTI, we aim to minimize the traveltime differences between the observed data $\mathbf{d}^{o}$ and the calculated data $\mathbf{d}^{c}$ using the following objective function:

$$
\left\{\begin{array}{l}
\tau\left(\mathbf{x}_{\mathbf{r}}, t\right)=\underset{\tau}{\arg \min } \int_{0}^{T} \sum_{r}\left\|\mathbf{d}^{c}\left(\mathbf{x}_{r}, t\right)-\mathbf{d}^{o}\left(\mathbf{x}_{r}, t+\tau\right)\right\|^{2} \\
E=\frac{1}{2} \int_{0}^{T} \sum_{r} \tau^{2}\left(\mathbf{x}_{\mathbf{r}}, t\right) \mathrm{d} t,
\end{array}\right.
$$

where the time-shift $\tau\left(\mathbf{x}_{\mathbf{r}}, t\right)$ can be extracted through DIW Hale (2013). For efficiency, we apply conventional RTM instead of its expensive least-squares counterpart to provide the perturbed stiffness coefficients because we focus on the kinematics.

After the derivation in Appendix A using the adjoint state method, the gradients of the objective function in eq. (4) can be expressed as

$\frac{\partial E}{\partial c_{i j k l}^{0}}=-\int\left(\frac{\partial u_{i}}{\partial x_{j}} \frac{\partial \hat{\psi}_{k}}{\partial x_{l}}+\frac{\partial \hat{u}_{i}}{\partial x_{j}} \frac{\partial \psi_{k}}{\partial x_{l}}\right) \mathrm{d} t$,

where $u_{i}$ and $\hat{u}_{i}$ are the state variables representing the forward background wavefields and perturbed wavefields, respectively. $\psi_{i}$ and $\hat{\psi}_{i}$ are the adjoint state variables representing the back-propagated background wavefields and the perturbed wavefields and satisfying

$\rho \frac{\partial \psi_{i}^{2}}{\partial t^{2}}-\frac{\partial}{\partial x_{j}}\left[c_{i j k l}^{0} \frac{\partial \psi_{k}}{\partial x_{l}}\right]=\tau\left(\mathbf{x}_{\mathbf{r}}, t\right) \frac{\dot{\mathrm{d}}_{i}^{o}\left(\mathbf{x}_{\mathbf{r}}, t+\tau\right)}{h\left(\mathbf{x}_{\mathbf{r}}, t\right)}$,

and

$\rho \frac{\partial \hat{\psi}_{i}^{2}}{\partial t^{2}}-\frac{\partial}{\partial x_{j}}\left[c_{i j k l}^{0} \frac{\partial \hat{\psi}_{k}}{\partial x_{l}}\right]=\frac{\partial}{\partial x_{j}}\left[c_{i j k l}^{1} \frac{\partial \psi_{k}}{\partial x_{l}}\right]$,

where $\quad h\left(\mathbf{x}_{\mathbf{r}}, t\right)=\dot{\mathbf{d}}^{o}\left(\mathbf{x}_{\mathbf{r}}, t+\tau\right)^{2}-\ddot{\mathbf{d}}^{o}\left(\mathbf{x}_{\mathbf{r}}, t+\tau\right)\left[\mathbf{d}^{c}\left(\mathbf{x}_{\mathbf{r}}, t\right)-\right.$ $\left.\mathbf{d}^{o}\left(\mathbf{x}_{\mathbf{r}}, t+\tau\right)\right]$ (the hat dot denotes the time derivative). The first equation indicates that the adjoint background wavefields $\psi$ is determined by the adjoint source at the receiver locations, whereas the second equation shows that the adjoint perturbed wavefield $\hat{\psi}$ is determined by the virtual source related to the high-wavenumber image perturbations. On the right-hand side of eq. (5), the two cross-correlations constitute the source and receiver parts of the reflection wave path.

For the isotropic case, the stiffness tensor satisfies

$c_{i j k l}=\rho\left(V_{\mathrm{p}}^{2}-2 V_{\mathrm{s}}^{2}\right) \delta_{i j} \delta_{k l}+\rho V_{\mathrm{s}}^{2}\left(\delta_{i k} \delta_{j l}+\delta_{i l} \delta_{j k}\right)$,

where $\delta_{i j}$ is the Kronecker symbol. For simplicity, we derive the gradients with respect to the stiffness coefficients for inversion, 


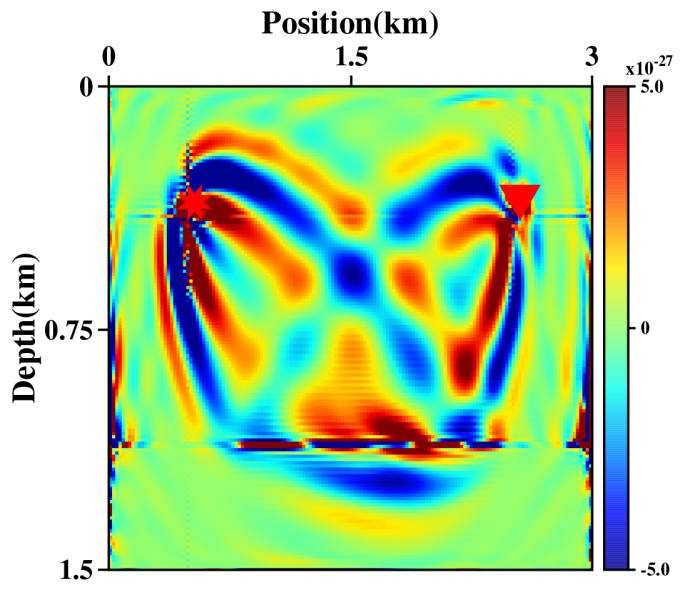

(a)

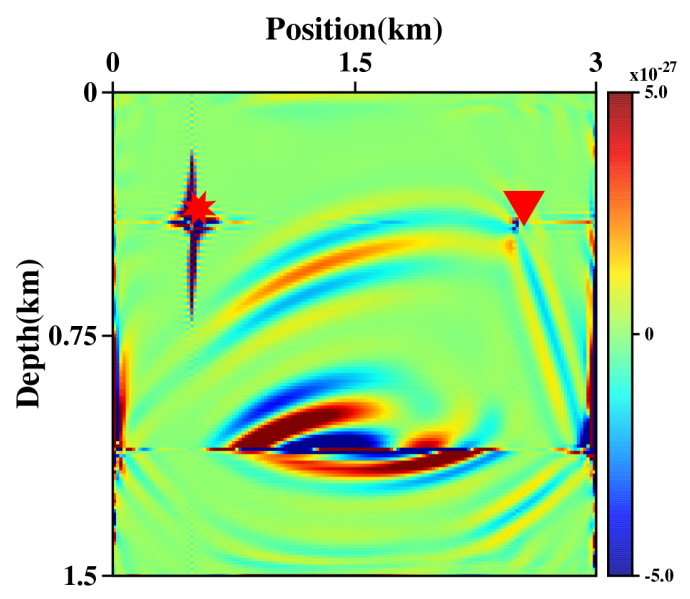

(c)

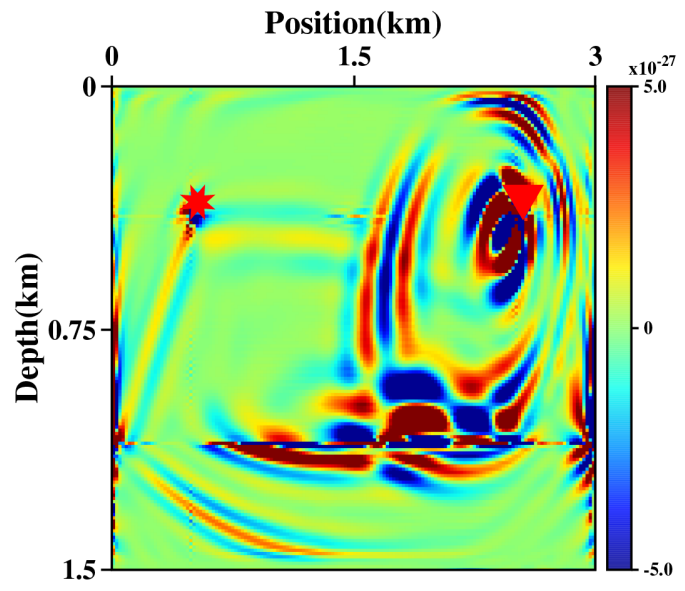

(b)

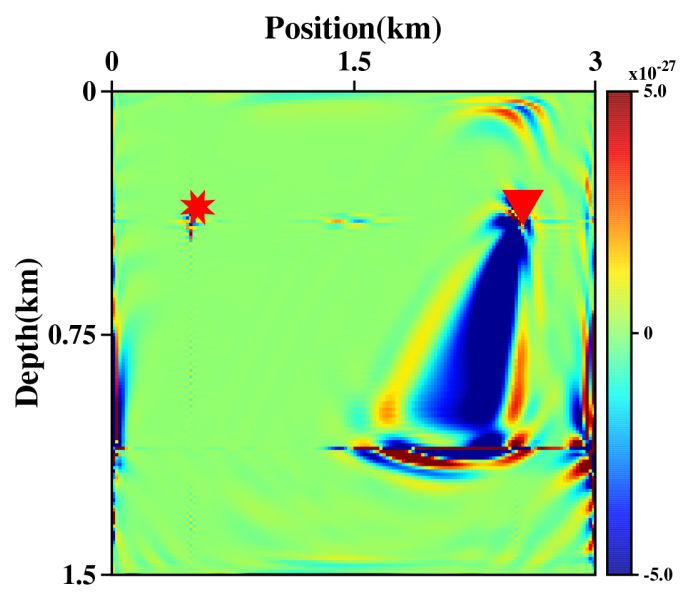

(d)

Figure 4. Four components of $K_{V_{\mathrm{s}}}$ : (a) $K_{V_{\mathrm{S}}}^{P P}$, (b) $K_{V_{\mathrm{S}}}^{P S}$, (c) $K_{V_{\mathrm{s}}}^{S P}$ and (d) $K_{V_{\mathrm{s}}}^{S S}$. Here, we only decompose the extrapolated elastic wavefields but do not apply the $P / S$ separation before injecting the adjoint sources on the recording surface.

however, the velocity parametrization is more reasonable for implementing the traveltime inversion. Thus, we obtain the gradients in terms of the background $V_{\mathrm{p}}$ and $V_{\mathrm{s}}$ through the chain rule, namely

$$
\begin{aligned}
& \frac{\partial E}{\partial V_{\mathrm{p}}}=2 \rho V_{\mathrm{p}} \frac{\partial E}{\partial c_{i j k l}^{0}} \delta_{i j} \delta_{k l}, \\
& \frac{\partial E}{\partial V_{\mathrm{s}}}=2 \rho V_{\mathrm{s}} \frac{\partial E}{\partial c_{i j k l}^{0}}\left(-2 \delta_{i j} \delta_{k l}+\delta_{i k} \delta_{j l}+\delta_{i l} \delta_{j k}\right) .
\end{aligned}
$$

\section{DECOMPOSITION OF ELASTIC REFLECTION KERNELS}

As demonstrated in Tromp et al. (2005) and Fichtner et al. (2006a,b), much of our physical intuition is based on the interpretation of sensitivity or Frechét kernels, which are defined as the volumetric densities of the misfit Frechét derivatives. Frechét kernels reveal how the objective function is affected by infinitesimal changes in the model parameters. The study of the reflection (Frechét) kernels enables the design of efficient inversion schemes and a physically meaningful interpretation of the results.
For brevity, we use a simple formula to express the gradient (or reflection kernel):

$\nabla E\left(\mathbf{m}_{0}\right)=-\int(\mathbf{u} \otimes \hat{\boldsymbol{\psi}}+\hat{\mathbf{u}} \otimes \boldsymbol{\psi})$

where $\mathbf{m}_{0}$ is the background model, $\mathbf{u}$ and $\hat{\mathbf{u}}$ are the incident and perturbed forward wavefields, and $\psi$ and $\hat{\psi}$ are the incident and perturbed adjoint wavefields, respectively. The operator $\otimes$ denotes the zero-lag cross-correlation between two wavefields. Note that eq. (10) just schematically shows the manner of cross-correlation. The detailed formula of a specified parametrization should be derived according to the chain rule as in eq. (9). Due to the complex wave phenomena, the wave path of elastic reflections is far more complicated than that in the acoustic case. Since the four wavefields in eq. (10) contain both $P$ - and $S$-waves, the cross-correlations between different wave mode conversions generate artefacts in the reflection kernel Wang et al. (2017b). In addition, the non-physical mode conversions occurring at the reflectors or recording surface further contaminate the reflection kernels. 
(a)
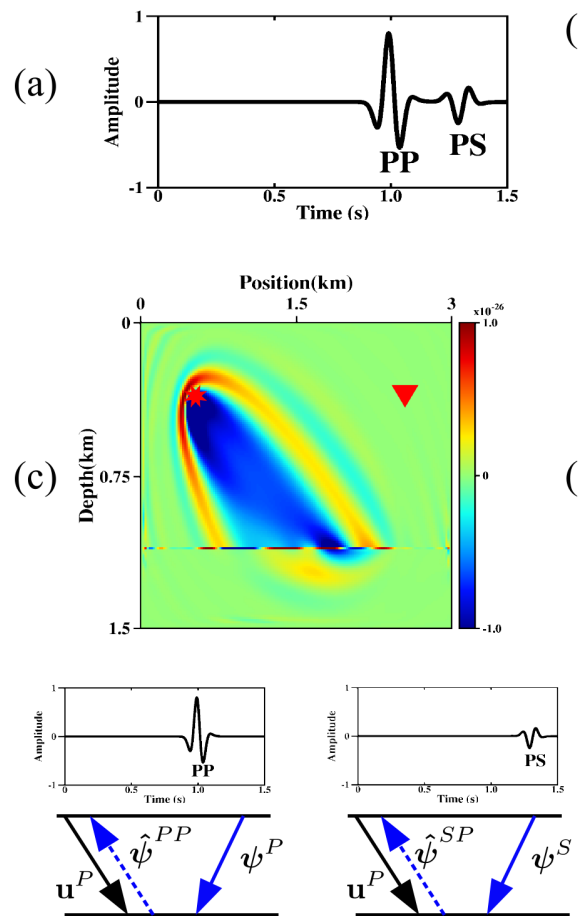

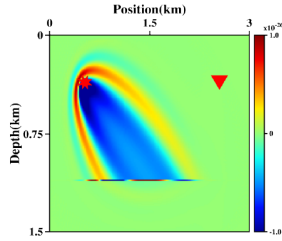

(e)

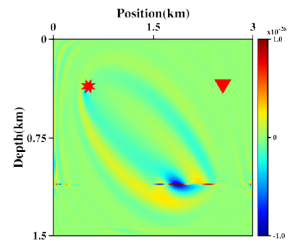

(f) (b)

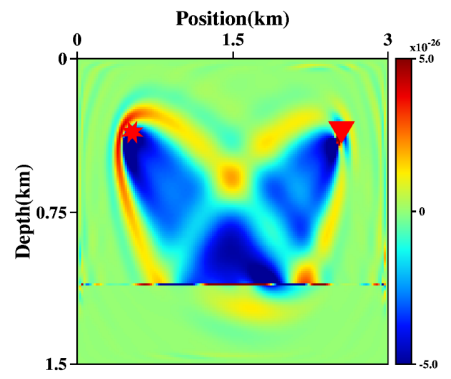

(d)
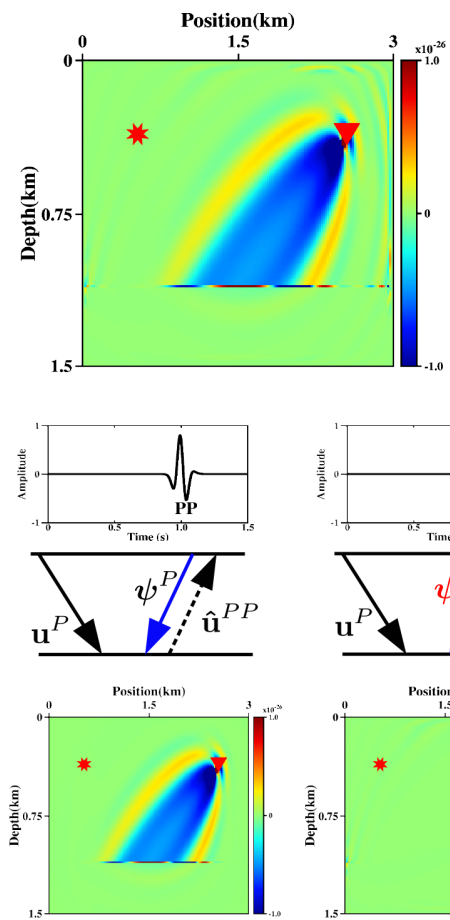

(g)
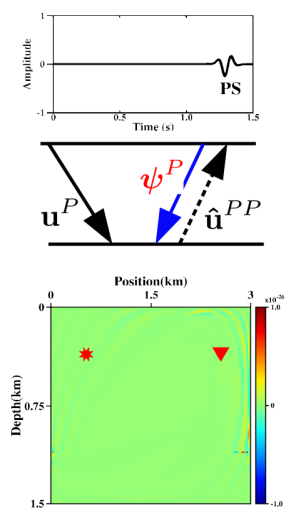

(h)

Figure 5. Decomposition of $K_{V_{\mathrm{p}}}$ in Fig. 3(c) by injecting the $P P$ and $P S$ events separately and decomposing the extrapolated elastic wavefields: (a) adjoint sources for backward-propagated wavefields; (b) $K_{V_{\mathrm{p}}}$, and its (c) source- and (d) receiver-side subkernels; panels (e)-(h) represent source- and receiver-side kernel components: the first and second rows denote the injected adjoint sources and the involved wave modes, while the last row shows the four kernel components. Note that the reflection kernels of the background $P$-wave velocity only result from common-mode cross-correlation of $P$-wave fields because of the divergence operations in eq. (9). Panel (h) displays some high-wavenumber artefacts very far away from the first Fresnel zone due to the non-physical mode conversions when injecting the $P S$ event at the receiver location.

To gain more insight into the elastic reflection kernel, we decompose it into four components, which correspond to the crosscorrelation of different wave modes, as follows:

$K_{m_{0}}^{M N}=-\int\left(\mathbf{u}^{M} \otimes \hat{\psi}^{N}+\hat{\mathbf{u}}^{M} \otimes \boldsymbol{\psi}^{N}\right), M, N \in\{P, S\}$.

$K_{m_{0}}^{M N}$ represents the sum of two cross-correlations between the forward wavefields of the $M$ mode and the adjoint wavefields of the $N$ mode. Note that the superscript $M$ or $N$ indicates the mode type of the wavefields involved in the cross-correlation rather than a particular wave mode in the seismograms.

As illustrated in Fig. 1(a), there is an $L$ mode incident wavefield that is converted to a $Q$ mode at the interface. Note $\overline{\mathbf{u}}$ represents the physical realization and $\mathbf{u}$ represents the numerically reconstructed wavefields in the subsurface. So, for a certain physical realization of the mode conversion (Fig. 1a), the corresponding migration or inversion process using the adjoint-state method may generate all kinds of wave modes during the wavefield extrapolation (Figs $1 \mathrm{~b}$ or c). Given the traveltimes $t_{1}$ and $t_{2}$ for the two legs of the ray path, the kernels calculated using eq. (11) can be considered as the results of pre-stack migration of the event recorded at the time $t_{1}+t_{2}$. Taking the source-side kernel as an example (Fig. 1b), the forward and adjoint wavefields will meet at an 'image' point with a 'scattering' angle of $\theta$, when $t\left(\mathbf{u}^{M}\right)+t\left(\hat{\boldsymbol{\psi}}^{N}\right)+t(\boldsymbol{\psi})=t_{1}+t_{2}$, where $t\left(\mathbf{u}^{M}\right)$ represents the traveltime of the forward background wavefields from source to the image point, $t\left(\hat{\psi}^{N}\right)$ represents the traveltime of the adjoint perturbed wavefields from the reflection point to the image point and $t(\boldsymbol{\psi})$ represents the traveltime of the adjoint background wavefields from the receiver to the reflection point. Like in the RTM results based on the cross-correlation imaging condition, the reflection kernels contain both low-wavenumber (transmission) and high-wavenumber (scattering) responses. When the 'scattering' angle approaching $180^{\circ}$ and $t\left(\mathbf{u}^{M}\right)+t\left(\hat{\psi}^{N}\right)=t_{1}$, the source-side leg becomes a transmission one, which admits near-zero wavenumber 
(a)
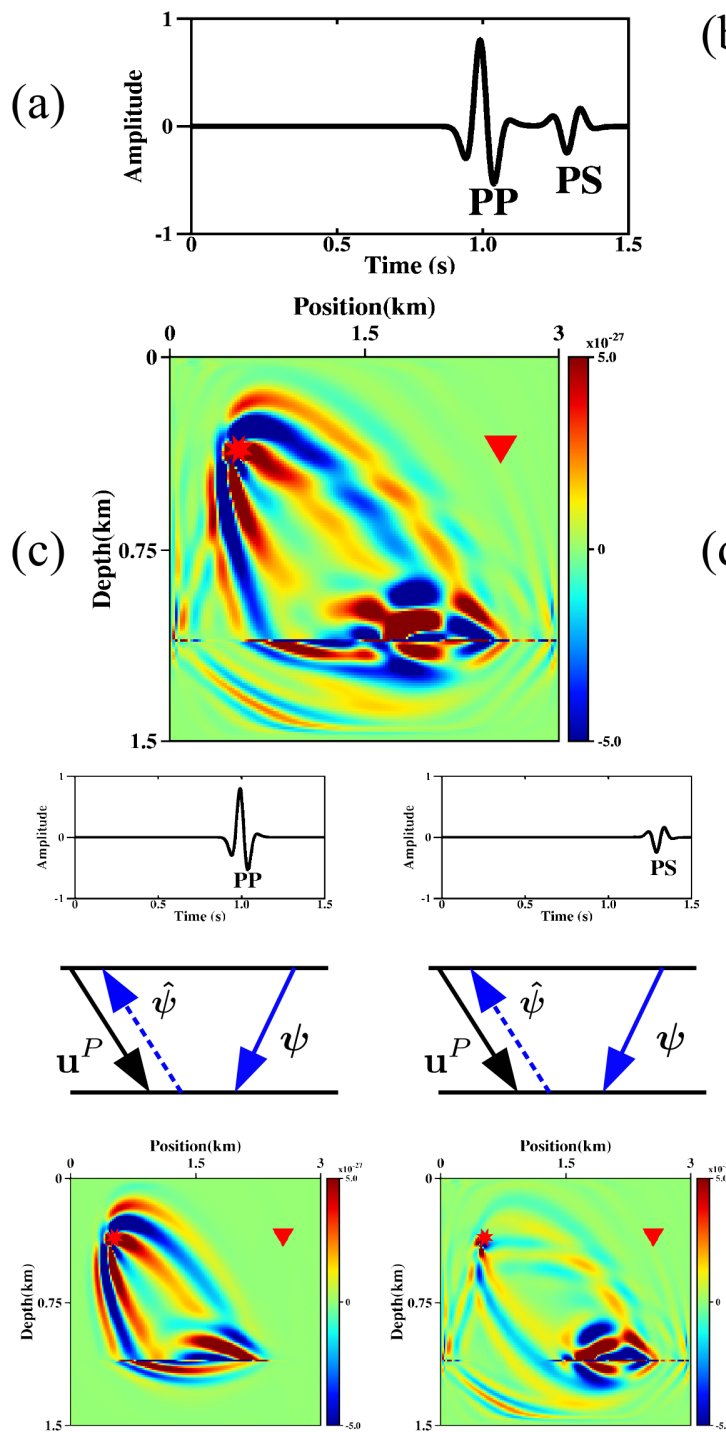

(e)

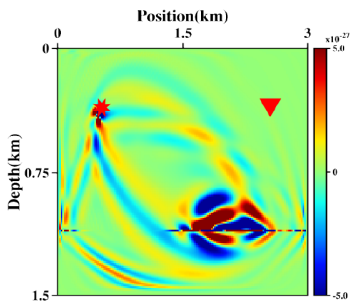

(f)

(b)
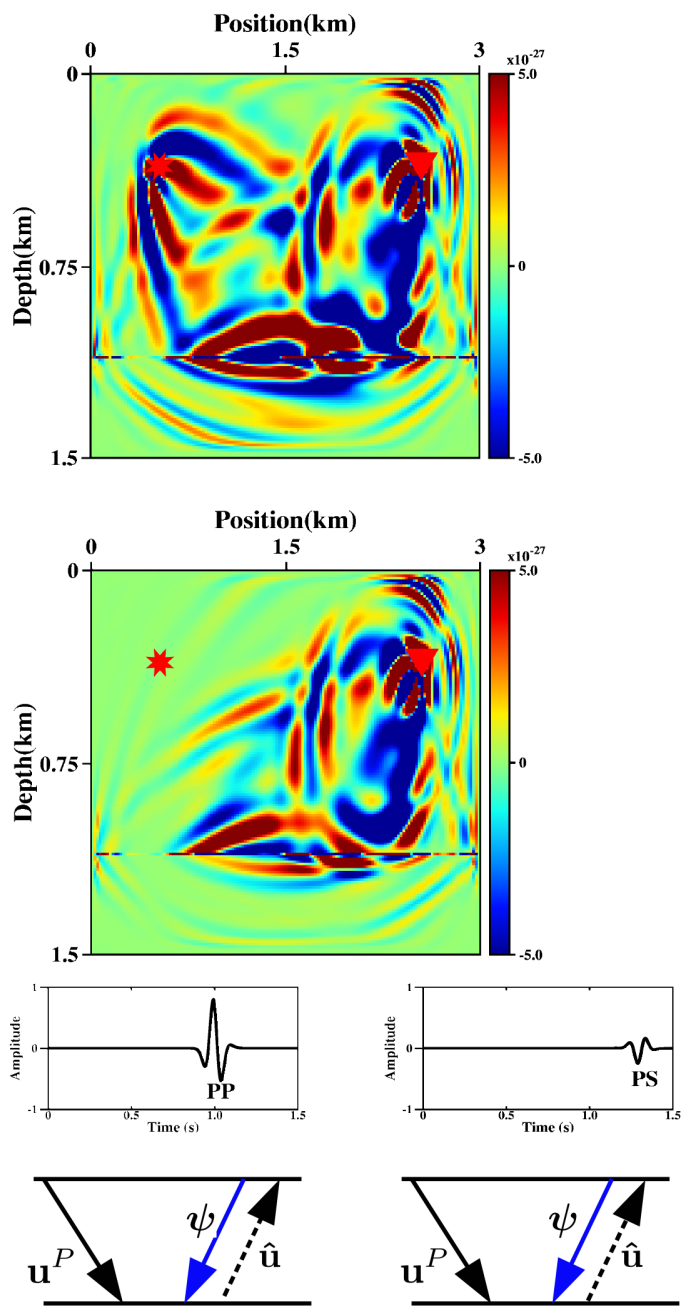

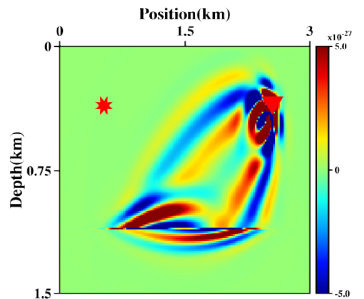

(g)

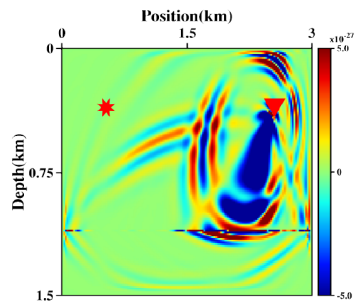

(h)

Figure 6. Decomposition of $K_{V_{\mathrm{S}}}$ in Fig. 3(d) by injecting the $P P$ and $P S$ events separately: (a) adjoint sources for backward-propagated wavefields; (b) $K_{V_{\mathrm{s}}}$, and its (c) source- and (d) receiver-side subkernels; panels (e)-(h) represent source- and receiver-side kernel components: the first row denotes the injected adjoint sources, the second row shows the involved wavefields and the bottom row shows the four kernel components. Note, this is only the first-level decomposition.

updates. Depending on the frequency, the wavenumber of the kernel will increase within the first Fresnel zone away from the ray path and yields reasonable resolution of the low-to-intermediate wavenumbers of the model. These components of the reflection kernel are required to build the background velocity models, while the others would be artefacts that have adverse effects on the inversion.

For illustration, we calculate the reflection kernels of a single source-receiver pair for a horizontal interface in a constant background. We use a pure $P$-wave source with a main frequency of $15 \mathrm{~Hz}$. True perturbation of the stiffness tensor is used to synthesize the perturbed wavefields. In the first test, the interface results from $V_{\mathrm{p}}$ perturbation at a depth of $1.1 \mathrm{~km}$ in a homogeneous medium
(Figs $2 \mathrm{a}$ and $\mathrm{b}$ ). Only $P P$ reflections exist in the data (like in acoustic media), so mode decomposition is not used in this case. As shown in Figs 2(c) and (d), the reflection kernels consist of twowide first Fresnel zones centred on the two-way ray paths between the reflection point and the source/receiver. $K_{V_{\mathrm{p}}}$ represents the lowwavenumber wave paths of the $P P$ reflection. In $K_{V_{\mathrm{s}}}$, we observe similar low-wavenumber wave paths that are relatively weaker at the centre of the first Fresnel zones, representing the effects of $P$-wave components on the reflection kernel of $V_{\mathrm{s}}$. As in acoustic reflection kernels Zhou et al. (2015), we also observe the isochrones located below the interface, which decrease in size at higher frequencies. 
(a)
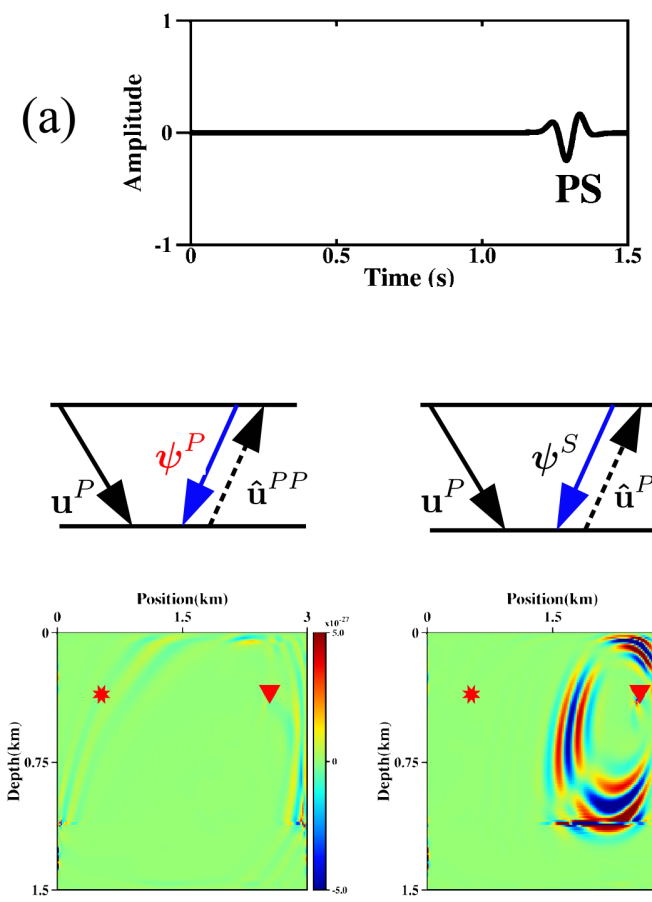

(c)
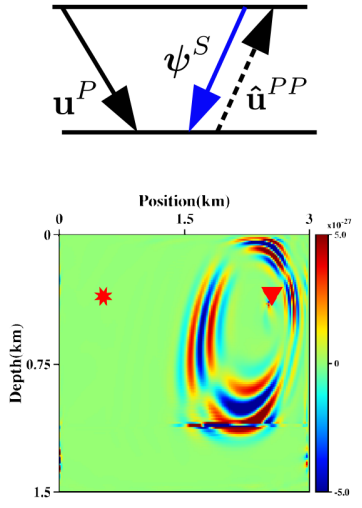

(d)

(b)
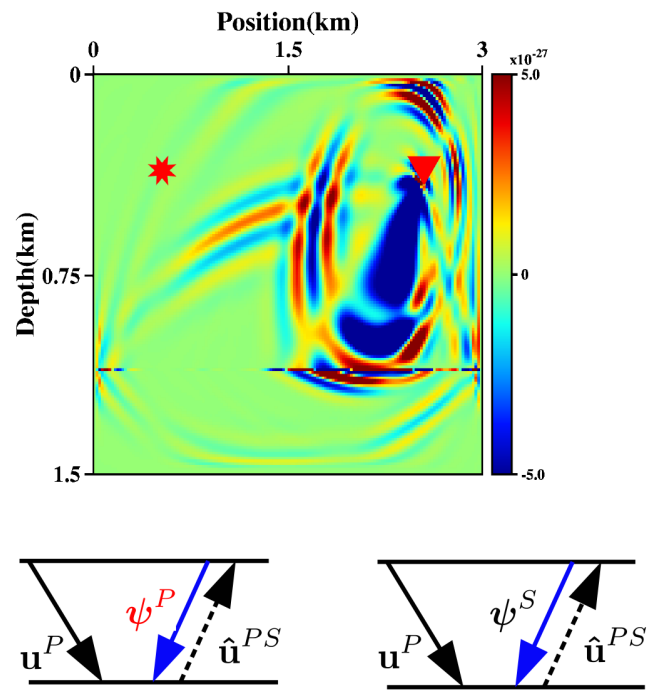

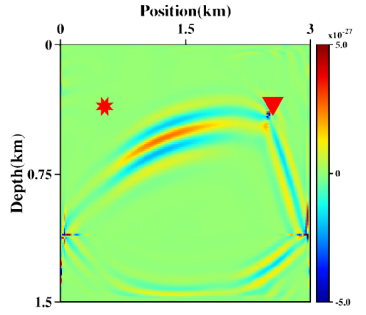

(e)

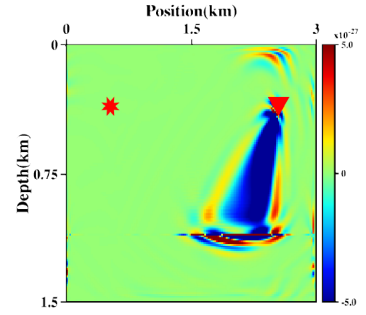

(f)

Figure 7. Further decomposition of the subkernel in Fig. 6(h) through mode decomposition of the extrapolated receiver-side forward (perturbed) and adjoint (background) wavefields: (a) The injected adjoint source; (b) subkernel of $K_{V_{\mathrm{s}}}$ to be decomposed; panels (c)-(f) represent the involved wave modes (top) and the corresponding kernel components (bottom). The red symbols $\left(\psi^{P}\right)$ in (c) and (e) denote the backward propagating $P$ wavefields resulting from the non-physical mode conversion at the receiver location. Note that only the common-mode cross-correlation of the $S$ wavefields in panel (f) corresponds to the reflection kernel because the background $S$-wave velocity only has effects on the receiver-side kinematics of $P S$ reflections.

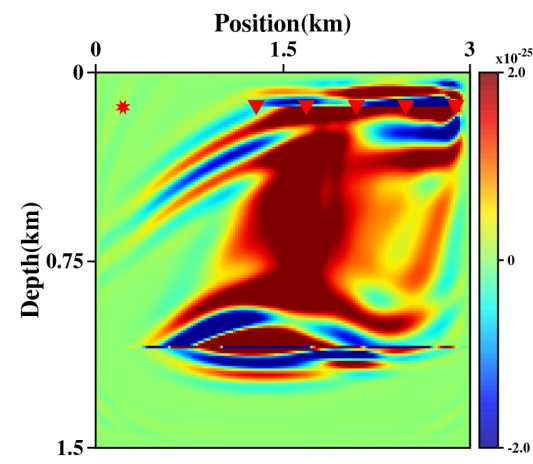

(a)

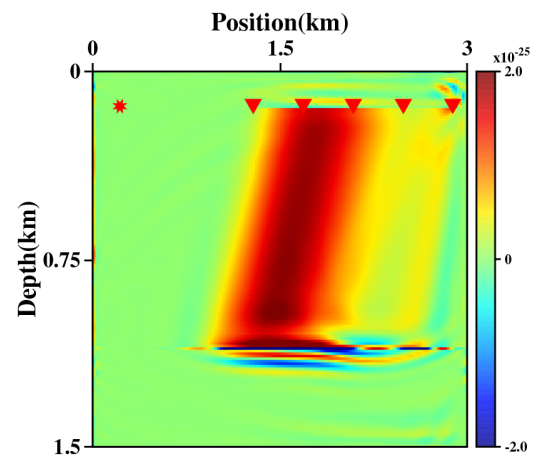

(b)

Figure 8. The gradient with respect to $V_{\mathrm{S}}$ with using the same model as in Fig. 3 but for a common-shot record: the source is on the left while the receivers are located on the top of the model with a minimal offset of $500 \mathrm{~m}$. (a) no $P / S$ separation on the recording surface or in the extrapolated wavefields, (b) using the proposed two-level mode decomposition method.

In the second test, the interface results from $V_{\mathrm{s}}$ perturbation (Figs $3 \mathrm{a}$ and $\mathrm{b}$ ) to generate both $P P$ and $P S$ reflections. The kernel of $V_{\mathrm{p}}$ excludes $S$-wave components automatically owing to the divergence operation implied in the gradient calculation, see eq. (9). However, the result is slightly different from that in Fig. 2(c) because mode conversions at the interface introduce the effects of the induced $S P$ waves in the adjoint perturbed wavefields. Various mode conversions and wave path overlapping make the kernel of
$V_{\mathrm{s}}$ even more complicated (Fig. 3d). As shown in Fig. 4, we can decompose $K_{V_{\mathrm{s}}}$ into four subkernels with eq. (11). Note that $K_{V_{\mathrm{s}}}^{P P}$ appears similar to $K_{V_{\mathrm{p}}}^{P P}$ (Fig. $3 \mathrm{c}$ ) but with an opposite polarity and a hollow centre because the terms (with $\delta_{i j} \delta_{k l}$ ) of the divergence operation have opposite signs, see eq. (9). The common-mode subkernels $\left(K_{V_{\mathrm{s}}}^{P P}\right.$ and $\left.K_{V_{\mathrm{s}}}^{S S}\right)$ mainly contain low-wavenumbers in the first Fresnel zones along the reflection wave paths, whereas the crossmode subkernels $\left(K_{V_{\mathrm{s}}}^{P S}\right.$ and $\left.K_{V_{\mathrm{s}}}^{S P}\right)$ have many artefacts of relatively 


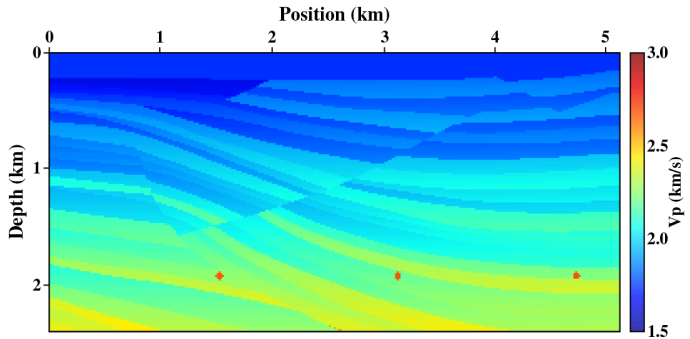

(a)

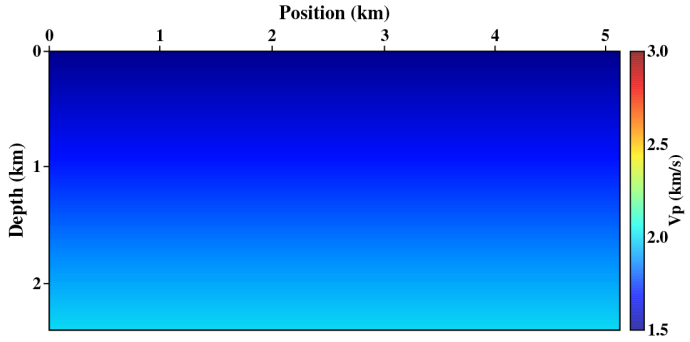

(c)

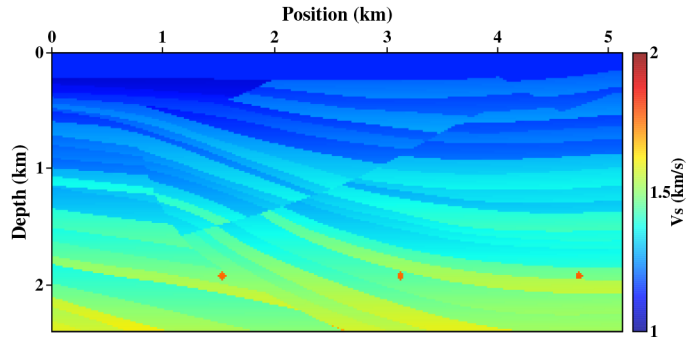

(b)

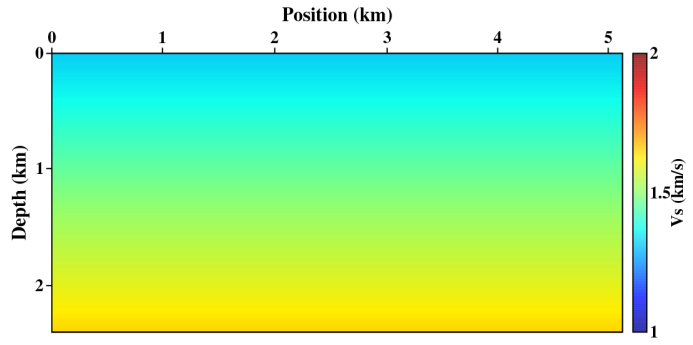

(d)

Figure 9. Sigsbee2A model example: on the top are the true models of $V_{\mathrm{p}}$ (a) and $V_{\mathrm{s}}$ (b), and at the bottom are their initial models for the migration and inversion experiments.

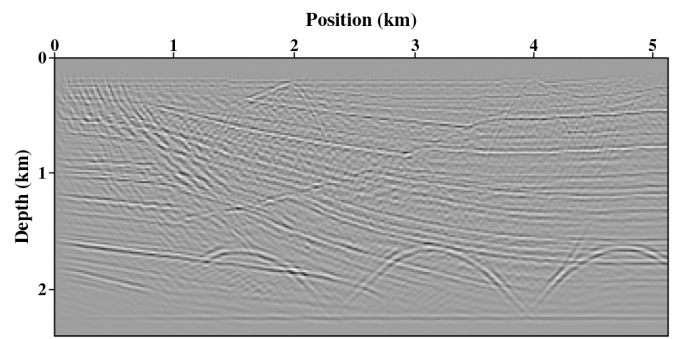

(a)

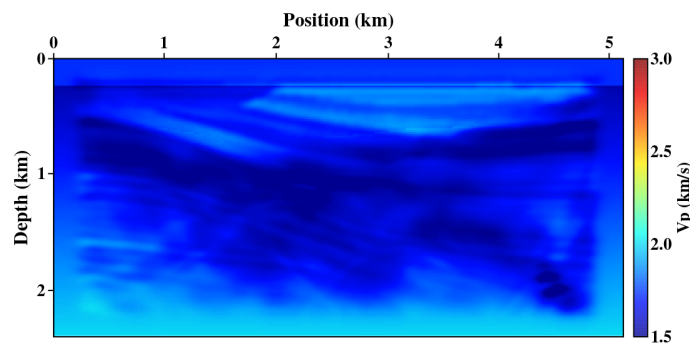

(c)

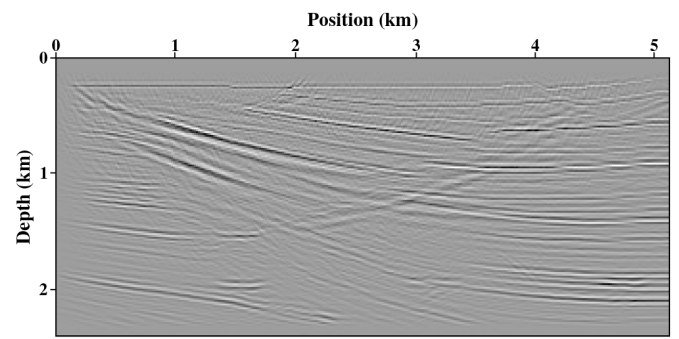

(b)

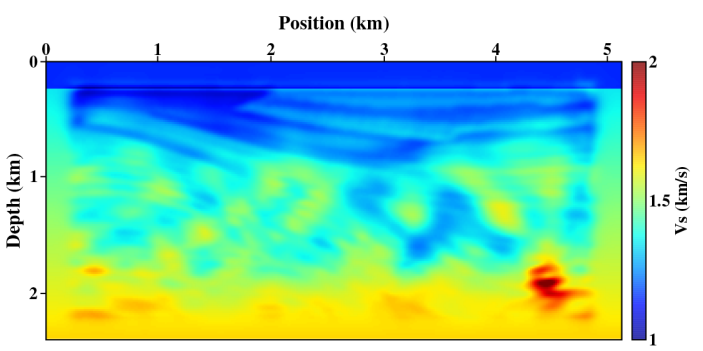

(d)

Figure 10. The results of ERTM (top) and EFWI (bottom) using the initial models: (a) $P P$ and (b) $P S$ images of near-offset data; (c) and (d) are the inverted $V_{\mathrm{p}}$ and $V_{\mathrm{s}}$ models.

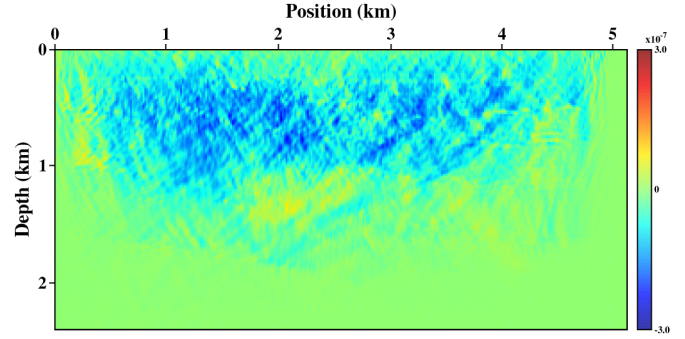

(a)

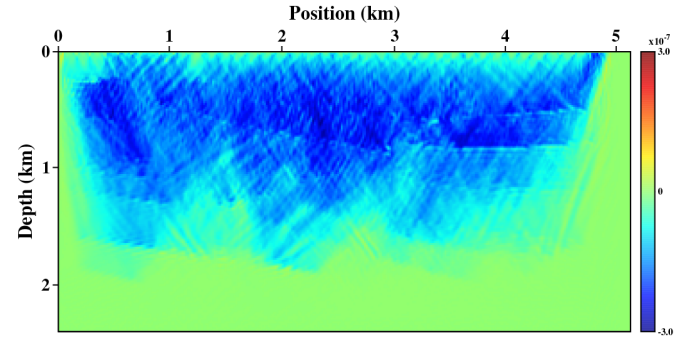

(b)

Figure 11. The gradients with respect to $V_{\mathrm{p}}$ calculated with (a) the original seismograms and (b) the isolated $P P$ seismograms. 


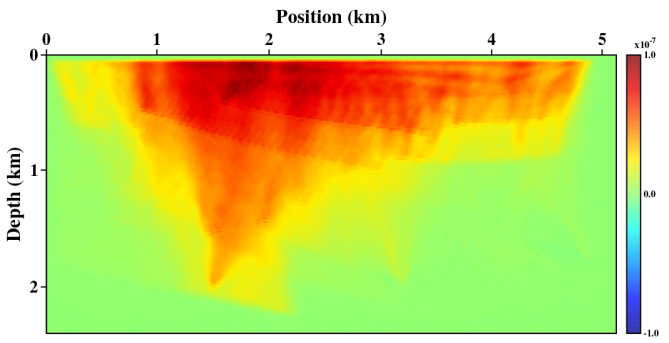

(a)

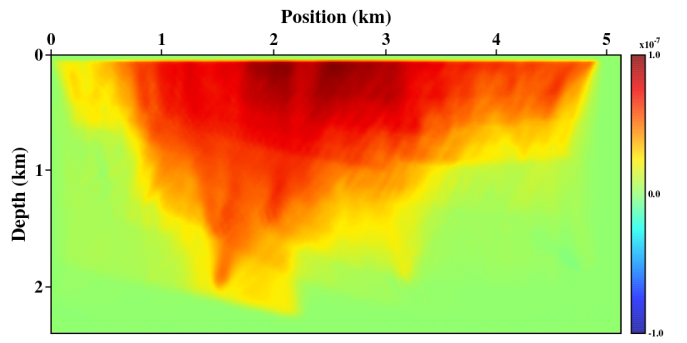

(b)

Figure 12. The gradients with respect to $V_{\mathrm{S}}$ (a) before and (b) after the application of mode-decomposition-based preconditioning.

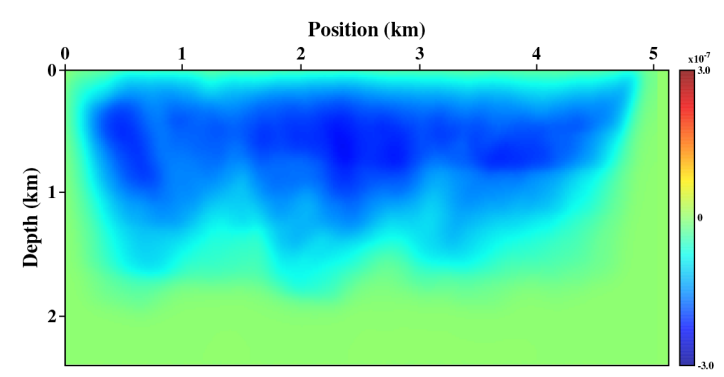

(a)

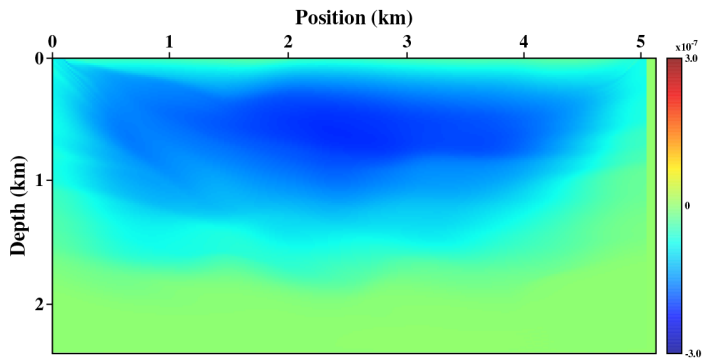

(b)

Figure 13. The gradients with respect to $V_{\mathrm{p}}$ (a) before and (b) after the structure-oriented regularization.

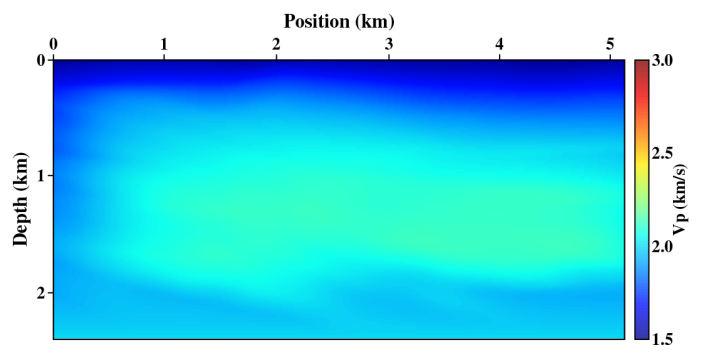

(a)

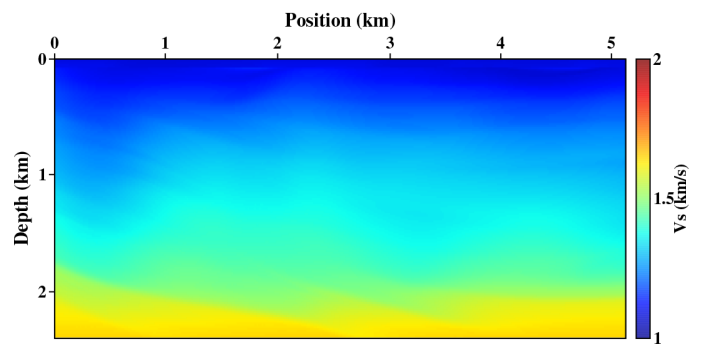

(b)

Figure 14. The inverted (a) $V_{\mathrm{p}}$ and (b) $V_{\mathrm{s}}$ models using ERTI. Due to the lack of reflection coverage, the background velocities close to the bottom cannot be reconstructed effectively.

higher wavenumbers, as well as some low wavenumbers very close to the mode conversion points.

Alternatively, we can decompose the reflection kernel by distinguishing the wave mode of the adjoint sources with $P / S$ separation of the residual seismograms on the recording surface. For the second test, we preferentially demonstrate the decomposed $K_{V_{\mathrm{p}}}$ (Fig. 5) because only $P$ waves participate in the cross-correlations due to the divergence operation on the extrapolated wavefields. Figs 5(e)(g) show the low-wavenumber common-mode subkernels along the reflection wave paths. The cross-correlation between the forwardperturbed $P P$ waves and the backward-propagated $S P$ waves from the non-physical mode conversions at the adjoint sources causes high-wavenumber artefacts far from the first Fresnel zone on the receiver side (Fig. 5h).

To gain a more physical understanding of the complicated reflection kernel of $V_{\mathrm{s}}$, we conduct the following two-level decomposition. First, we distinguish $P P$ and $P S$ events when injecting the adjoint sources. As shown in Fig. 6, the source- and receiver-side kernels are decomposed into two subkernels; then, these subkernels are further decomposed via mode decoupling of the extrapolated forward and adjoint wavefields before cross-correlation. As an example, Fig. 7 displays the decomposition of the subkernel in Fig. 6(h). When a
$P S$ event is injected as the adjoint sources, $S P$ modes $\left(\psi^{P}\right)$ exist in the adjoint background wavefields stemming from the non-physical mode conversions at the receiver locations. This leads to relatively high-wavenumber 'migration' responses (Figs $7 \mathrm{c}$ and e). Moreover, as shown in Figs 7(d) and (e), cross-mode cross-correlation also results in strong artefacts in the reflection kernel. Common mode cross-correlation between the forward-perturbed and adjoint background wavefields ( $\hat{\mathbf{u}}^{P S}$ and $\boldsymbol{\psi}^{S}$ ) generates low-wavenumber components along the receiver-side wave paths of the reflected $P S$ waves. The rest of the second-level decomposition process is discussed in Appendix B.

In physics, background $P$-wave velocities control the traveltime of $P P$ reflections, whereas background $S$-wave velocities determinate the receiver-side traveltimes of $P S$ reflections. Therefore, it is better to use the $P P$-wave path to recover the background $P$-wave velocity and use the receiver-side wave path of $P S$ events to recover the background $S$-wave velocity. Based on the above kernel decomposition, two kinds of components, the common-mode components related to non-physical conversions and the cross-mode components, represent artefacts in the gradients (or reflection kernels). Fig. 8 shows the contribution of seismogram $P / S$ separation and wavefield mode decomposition to the one-shot gradient with 


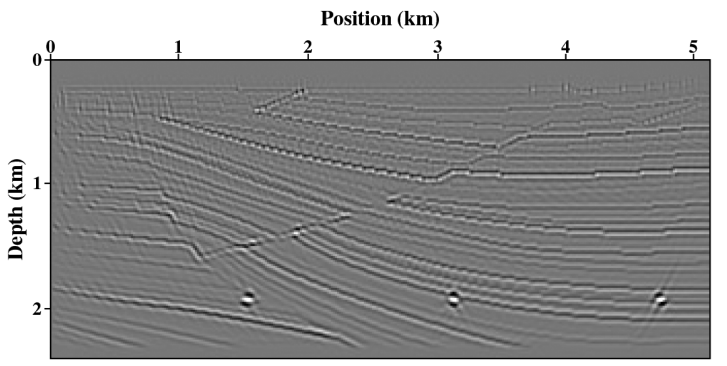

(a)

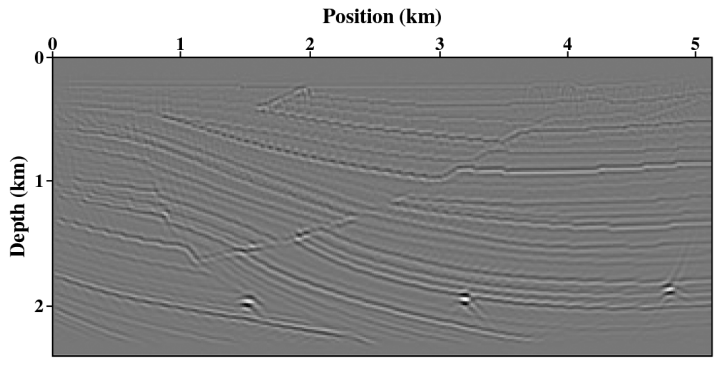

(c)

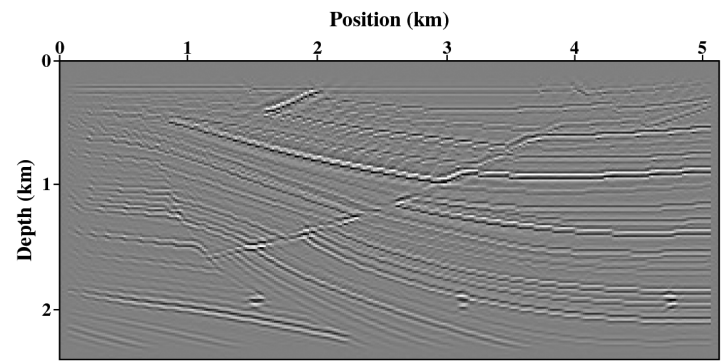

(b)

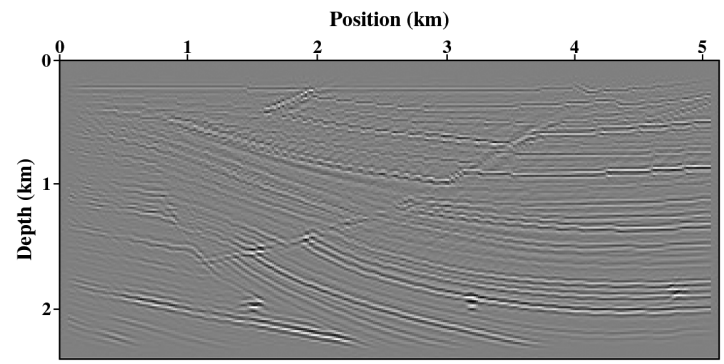

(d)

Figure 15. $P P$ (left) and $P S$ (right) images of ERTM using the true (top) and inverted (bottom) models.

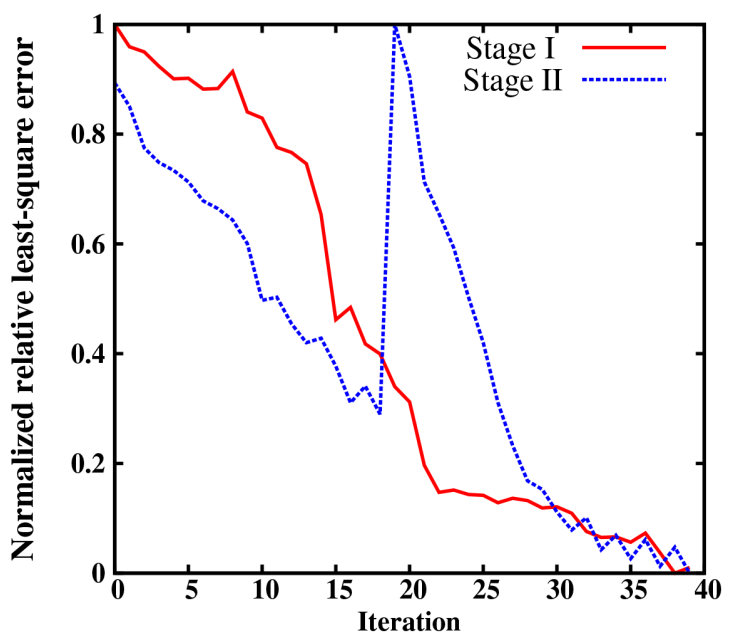

Figure 16. Normalized relative least-squares error of $P P$ and $P S$ traveltime in terms of iterations. The red line indicates $P P$ data while blue line indicates $P S$ data. Since we used the layer-stripping strategy in the Stage II, the misfit of $P S$ traveltime presents a peak when we include all the data at the 20th iteration.

respect to $V_{\mathrm{s}}$. Although multifold stacking can reduce part of the artefacts, obvious high-wavenumber energy still remains in the gradient. Moreover, the gradient in Fig. 8(a) also contain the $P$-wave path which may mislead the recovery of $V_{\mathrm{s}}$, while our proposed method gives quite smooth and clean gradient.

Thus, separating the $P P$ and $P S$ data when injecting the adjoint sources and decoupling the $P$ - and $S$-wavefields before crosscorrelation is helpful to suppress these artefacts. For instance, the reflection kernel of $V_{\mathrm{p}}$ is well represented if we calculate it only with the isolated $P P$ event, see Fig. 5. Even in the other common-mode components, however, $P$-wave related cross-correlations lead to similar wave paths, but with opposite polarities, for the background
$V_{\mathrm{p}}$ and $V_{\mathrm{s}}$. Similar phenomena are also mentioned by (Wang \& Cheng 2017, Fig. 7) in the EFWI problem. Therefore, the gradienttype linearized optimization still suffers from the trade-off problem. For our interests, the common-mode component corresponding to the cross-correlation of $S$-waves adequately represents the receiverside wave path of the isolated $P S$ event. Therefore, we recommend using this component to mitigate the trade-offs and to suppress the artefacts when recovering the background $V_{\mathrm{s}}$ model.

\section{WORKFLOW OF WAVE-EQUATION-BASED ERTI}

Multicomponent seismograms generally contain records of different wave modes corresponding to various discontinuity points and/or interfaces in the subsurface media. Even in the recorded data of $P$ wave sources, we often observe both $P P$ and $P S$ reflections, which makes it more challenging to measure the traveltime misfits between synthetic and recorded seismograms than in acoustic cases. However, the kernel decomposition tells us that distinguishing the wave modes of adjoint sources provides an opportunity to reduce the artefacts in the gradient calculation. Therefore, we apply $P / S$ separation of multicomponent seismograms Li et al. (2016) for easier estimation of the time-shifts through DIW and better improvements of the gradients. Accordingly, we propose an ERTI approach through a two-stage workflow: estimate $V_{\mathrm{p}}$ using $P P$ reflections and then estimate $V_{\mathrm{s}}$ using $P S$ reflections.

\subsection{Stage I: ERTI of $P P$ reflection}

In this stage, we use the traveltimes of $P P$ reflection to recover the low-to-intermediate wavenumbers of the background $V_{\mathrm{p}}$ model. The $P P$ image acts as the perturbation of $V_{\mathrm{p}}$, that is, $\delta V_{\mathrm{p}}$, which represents the secondary sources to generate the synthetic reflection data. When the migration velocity model is inaccurate, the two-way 


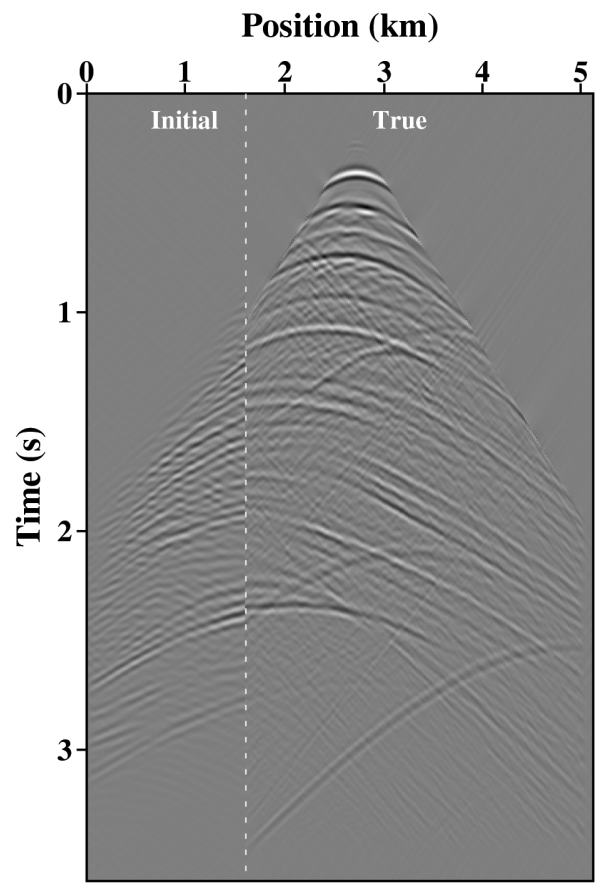

(a)

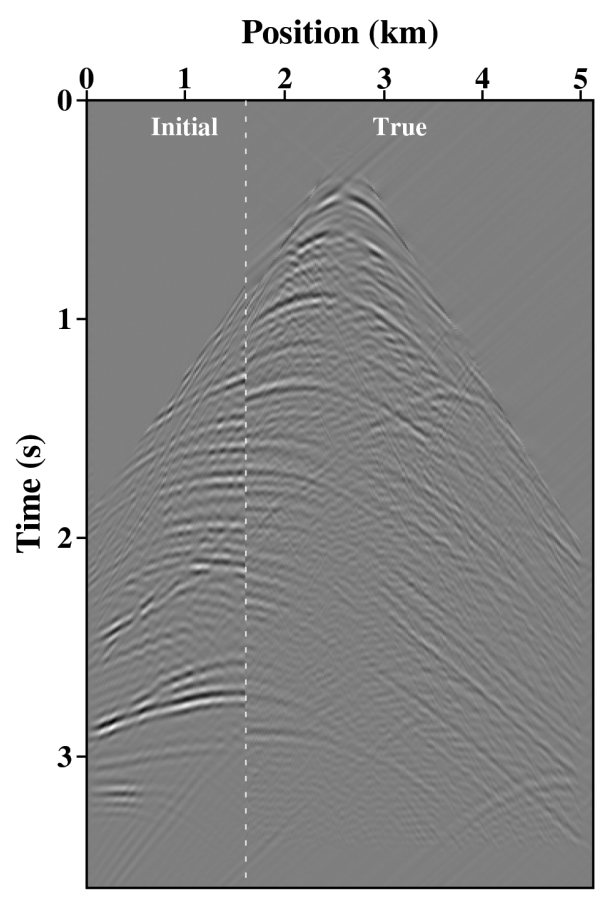

(c)

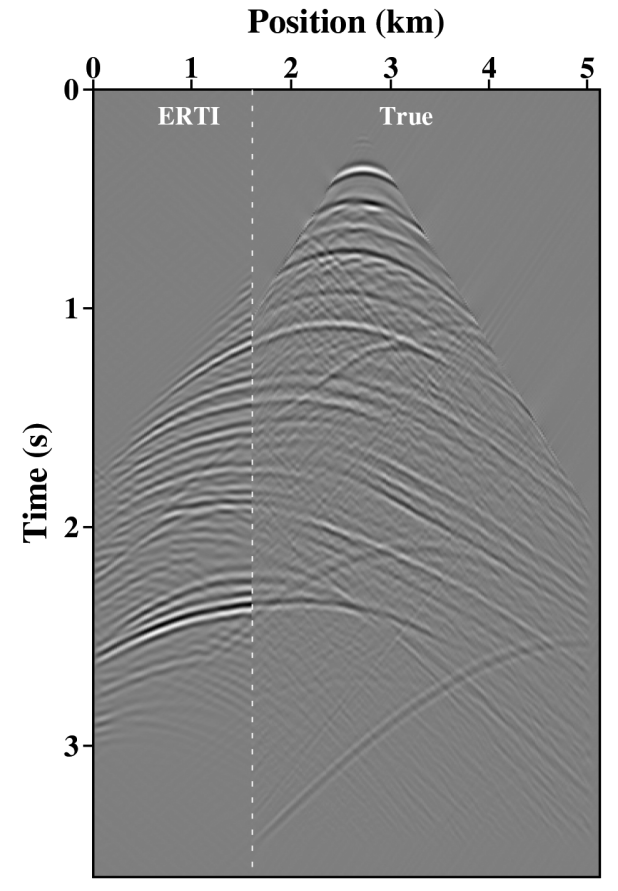

(b)

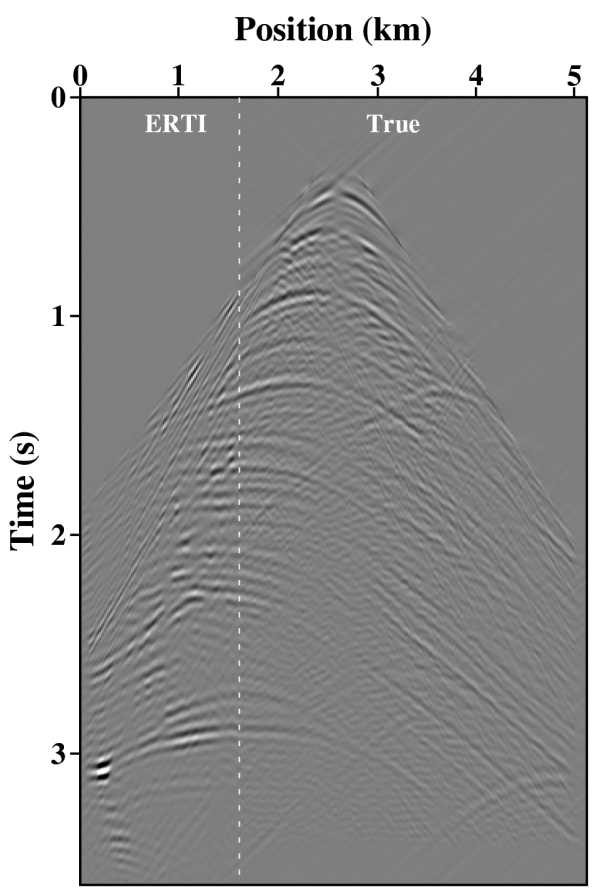

(d)

Figure 17. Comparison of the predicted and recorded $P P$ and $P S$ seismograms. The demigrated reflection data (left part of the dash line) are tailored together with the observed data (right part of the dash line). (a) $P P$ seismograms with initial and true model, (b) $P P$ seismograms with ERTI and true model, (c) $P S$ seismograms with initial and true model and (d) $P S$ seismograms with ERTI and true model.

traveltime of the zero-offset is taken as invariant during reflection inversion. As in the process of RWI, for example, Zhou et al. (2015), a zero- or small-offset image is used to provide the velocity perturbation for demigration. When the velocity anomaly is complex, a better method is the demigration of the extended image Weibull \&
Arntsen (2014); Hou \& Symes (2015); Guo et al. (2017), but it is out of the scope of this paper.

Since traveltimes rather than amplitudes are fitted in ERTI, elastic reverse time migration (ERTM; instead of its least-squares counterpart) is sufficient to obtain the image perturbation for demigration. 


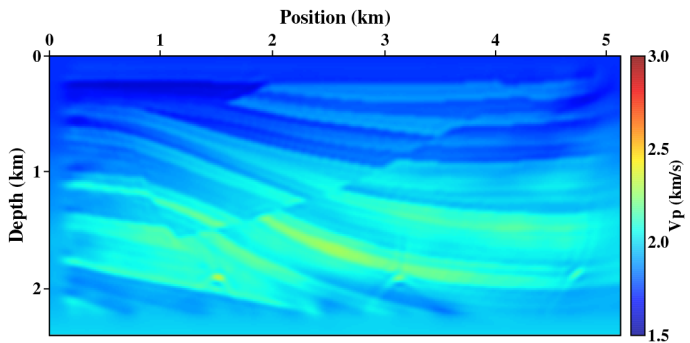

(a)

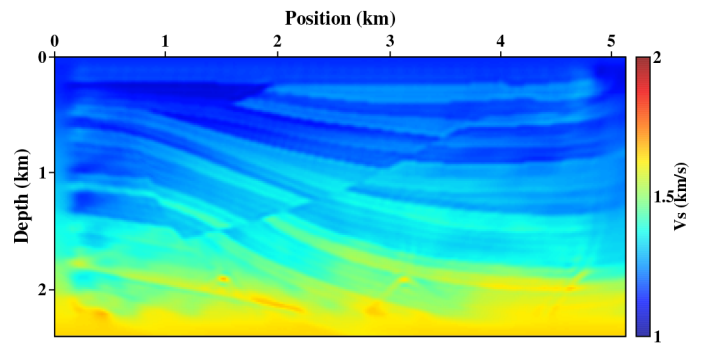

(b)

Figure 18. EFWI results using the inverted models of ERTI as the starting models: (a) $V_{\mathrm{p}}$ and (b) $V_{\mathrm{s}}$.
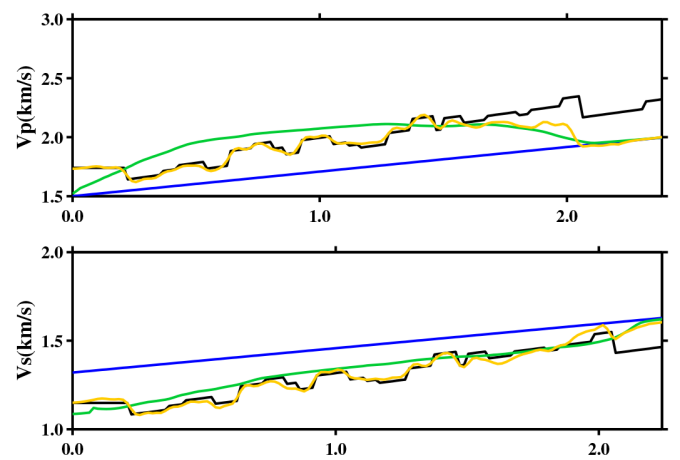

(a)
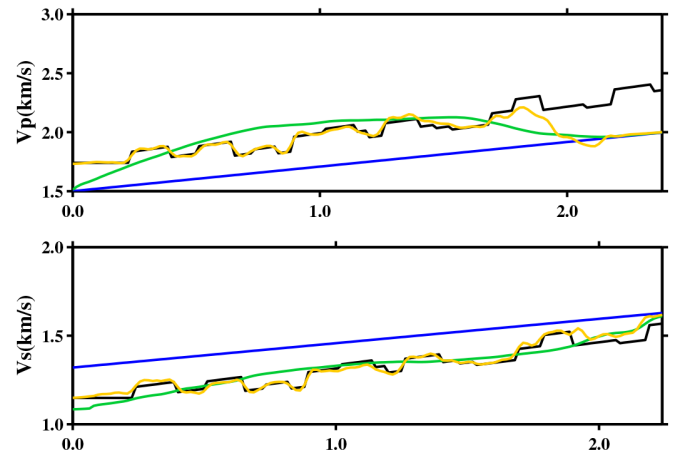

(b)

Figure 19. Vertical profiles of the inverted models obtained through application of ERTI (green) and EFWI (yellow) in sequence: (a) $x=1.4 \mathrm{~km}$ and (b) $x=$ $3.0 \mathrm{~km}$. The black and blue lines denote the true and initial models of $V_{\mathrm{p}}$ (top) and $V_{\mathrm{s}}$ (bottom).

Thus, the objective function is specified as

$$
\left\{\begin{array}{l}
\tau_{p p}\left(\mathbf{x}_{\mathbf{r}}, t\right)=\underset{\tau}{\arg \min }\left\|\mathbf{d}_{p p}^{c}\left(\mathbf{x}_{r}, t\right)-\mathbf{d}_{p p}^{o}\left(\mathbf{x}_{r}, t+\tau\right)\right\|^{2} \\
E_{p p}=\frac{1}{2} \int \tau_{p p}^{2}\left(\mathbf{x}_{\mathbf{r}}, t\right) \mathrm{d} t \mathrm{~d} \mathbf{x}_{\mathbf{r}},
\end{array}\right.
$$

where $\mathbf{d}_{p p}^{c}$ and $\mathbf{d}_{p p}^{o}$ are the synthesized and observed $P P$ reflections after $P / S$ separation on the recording surface, respectively. According to the previous derivation, the gradient with respect to $V_{\mathrm{p}}$, that is, $\frac{\partial E}{\partial V_{p}}$, satisfies the same expression as eq. (9), in which the background wavefields backward propagate from a slightly modified adjoint source. The divergence operation implied in $\frac{\partial E}{\partial V_{p}}$ makes the gradient calculation only involving common-mode cross-correlation of the $P$-waves in the regular and adjoint wavefields. In fact, the $P$-wave seismograms isolated through $P / S$ separation of multicomponent recordings may contain $S P$ components due to the elastic effects on the wave path of propagation. For simplicity, we ignore these components in this paper.

\subsection{Stage II: ERTI of $P S$ reflection}

In the second stage, we utilize the $P S$ reflections to retrieve the background $V_{\mathrm{s}}$ model. The objective function becomes

$$
\left\{\begin{array}{l}
\tau_{p s}\left(\mathbf{x}_{\mathbf{r}}, t\right)=\underset{\tau}{\arg \min }\left\|\mathbf{d}_{p s}^{c}\left(\mathbf{x}_{r}, t\right)-\mathbf{d}_{p s}^{o}\left(\mathbf{x}_{r}, t+\tau\right)\right\|^{2} \\
E_{p s}=\frac{1}{2} \int \tau_{p s}^{2}\left(\mathbf{x}_{\mathbf{r}}, t\right) \mathrm{d} t \mathrm{~d} \mathbf{x}_{\mathbf{r}},
\end{array}\right.
$$

where $\mathbf{d}_{p s}^{c}$ and $\mathbf{d}_{p s}^{o}$ are the synthesized and observed $P S$ reflections, respectively. According to our investigation, the lack of zero-offset components leads to abnormal reconstruction of the kinematics for the near-offsets through demigration of PS images. Because both the background and perturbation (i.e. imaged interfaces) of $V_{\mathrm{p}}$ are well recovered in the first stage, and in most geological settings, $V_{\mathrm{p}}$ and $V_{\mathrm{s}}$ have consistent structures in the subsurface; therefore, we can use the well-located interfaces of the first stage to generate the $P S$ reflections for reliable kinematic information.

During this stage, we use the well-recovered background $V_{\mathrm{p}}$ model built from Stage I and the initial $V_{\mathrm{s}}$ model as input. The $P P$ image is used as perturbation of $V_{\mathrm{s}}$ after some depth-related normalizations and remains unchanged in this stage. Therefore, the accumulated traveltime residuals of the demigrated $P S$ events may be large in the deeper part due to overburden velocity errors. DIW may suffer from cycle skipping under these conditions. Note, in Stage I, DIW does not have such problems because we use the nearoffset $P P$ image to generate $P P$ reflections. To address this problem, we utilize the 'layer-stripping' strategy, in which the shallow part is recovered using the early-arrived reflections, whereas the deep part is gradually recovered using the late-arrived reflections. In this way, the incorrect traveltime residuals of the deeper part will not negatively affect the inversion of the shallow part. Moreover, reliable inversion of the shallow part guarantees the estimation of the traveltime residual for the deeper part.

Given the correct background $V_{\mathrm{p}}$ model, the traveltimes of the $P S$ events are sensitive to only the change of $V_{\mathrm{s}}$ along the receiver-side reflection wave path. To ensure that the reflected $S$-wave kernels are used to update the background $V_{\mathrm{s}}$ model, elastic wave mode decomposition is applied to calculate $K_{V_{\mathrm{s}}}^{S S}$, namely

$\frac{\partial E_{p s}}{\partial V_{\mathrm{s}}}=-2 \rho V_{\mathrm{s}} \int\left(\frac{\partial \hat{u}_{i}^{S}}{\partial x_{j}} \frac{\partial \psi_{k}^{S}}{\partial x_{l}}\right)\left(\delta_{i k} \delta_{j l}+\delta_{i l} \delta_{j k}\right)$.

This process is similar to the gradient preconditioner for EFWI proposed by Wang \& Cheng (2017), and it can suppress artefacts in 


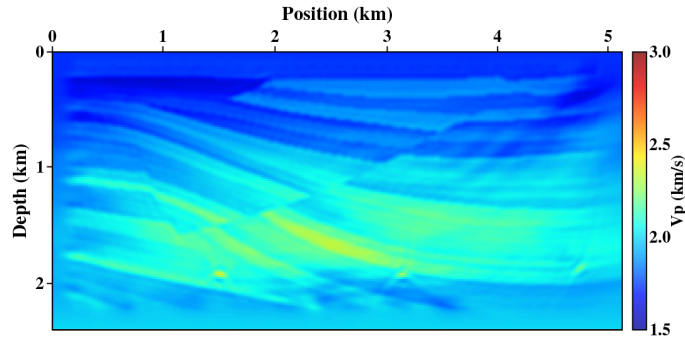

(a)

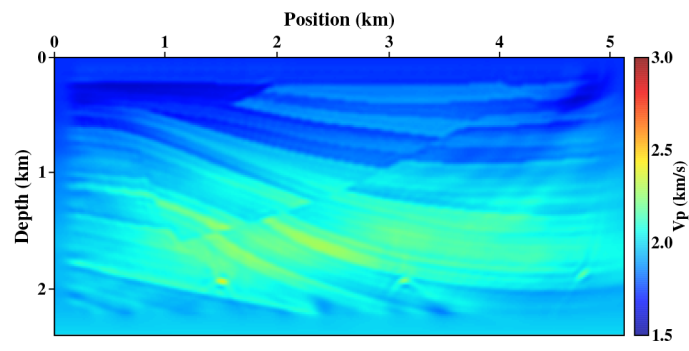

(c)

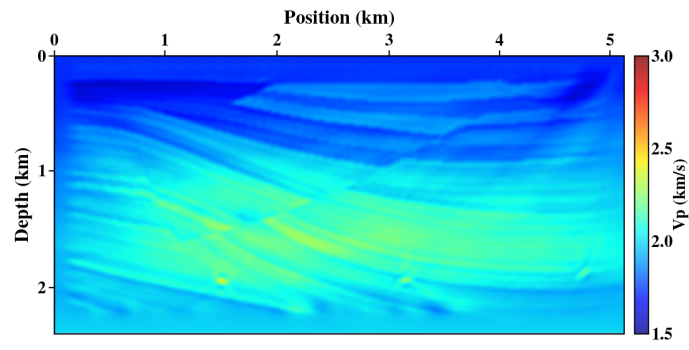

(e)

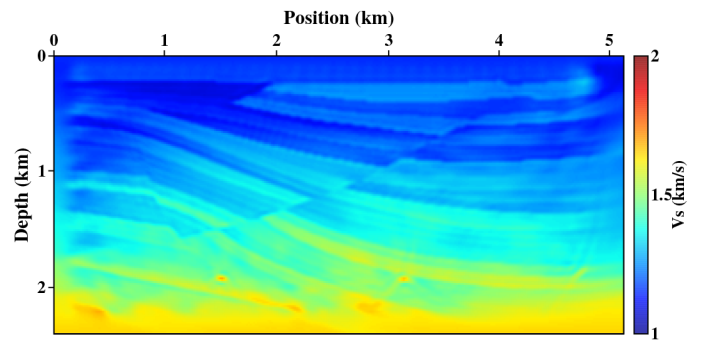

(b)

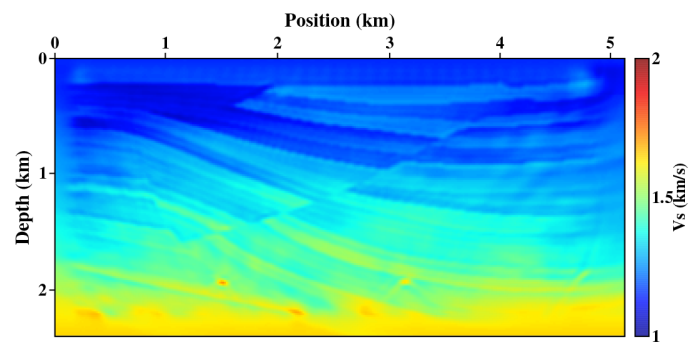

(d)

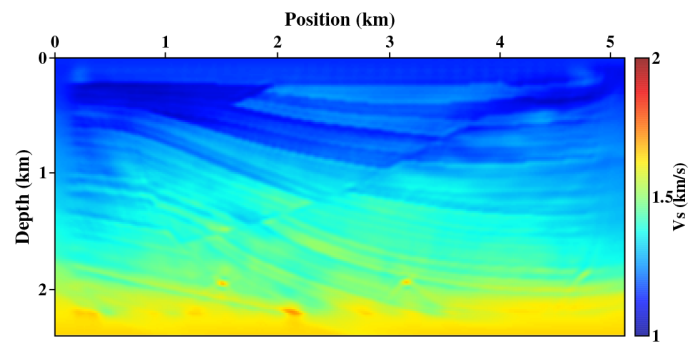

(f)

Figure 20. Recovered $V_{\mathrm{p}}$ (left) and $V_{\mathrm{S}}$ (right) by ERTI + EFWI with different high-pass filtering of the seismograms. The low-cut frequencies are $3.0,5.0$ and $7.0 \mathrm{~Hz}$ from top to bottom, respectively.

the gradients and mitigate parameter trade-offs for the inversion of $V_{\mathrm{s}}$.

\section{NUMERICAL EXAMPLE}

We select part of the Sigsbee2A model to test the inversion algorithm and strategy. As shown in Figs 9(a) and (b), the $V_{\mathrm{s}}$ model is generated through the $V_{\mathrm{p}}$ model with a constant $V_{\mathrm{s}}$-to- $V_{\mathrm{p}}$ ratio of 0.66 . The initial models for ERTI, which increase linearly with depth, are shown in Figs 9(c) and (d). The initial model of $V_{\mathrm{p}}$ is generally lower (from 1500 to $1996 \mathrm{~m} \mathrm{~s}^{-1}$ ) while that of $V_{\mathrm{s}}$ is higher (from 990 to $1317 \mathrm{~m} \mathrm{~s}^{-1}$ )) than the true ones. 48 shots and 320 receivers are evenly deployed on the surface. The main frequency of the $P$ wave source is $15 \mathrm{~Hz}$, and the spatial and time sampling intervals of the forward modelling are $16 \mathrm{~m}$ and $1.2 \mathrm{~ms}$, respectively.

Fig. 10 shows the results of ERTM and EFWI with the initial model. Since the initial models are far from the true ones, neither the $P P$ image nor the $P S$ image are well recovered with ERTM. The diffractions do not collapse in the PP image, and the faults are not focused and inappropriately positioned in the PS image. During EFWI, a hierarchical strategy is applied from low to high frequency through the time-domain low-pass filtering of the data. Thus, the inversion is divided into four stages in which the allowable maximum frequency are 2, 4, 6 and $8 \mathrm{~Hz}$, respectively. The shallow parts of the updated models are acceptable due to the contribution of diving waves and low-frequency data. However, the inversion of the deep part suffers from severe cycle skipping due to the absence of low-to-intermediate wavenumber components in the initial model.

Starting from the same initial model, we implement the proposed two-stage ERTI workflow. During the inversion, the direct waves are muted to ensure that only reflection data are used. In the first stage, the isolation of the $P P$ seismogram ensures good estimation of the $P P$ traveltimes and helps to reduce the artefacts caused by $P S$ events. Fig. 11 shows that the gradient with respect to $V_{\mathrm{p}}$ using the separated $P P$ seismograms contains more low wavenumbers than that using the original seismograms. In the second stage, mode decomposition-based preconditioning remarkably improves the gradient with respect to the background $S$-wave velocity (Fig. 12), which helps to effectively update the background velocity models along the reflection wave paths in a large spatial range. In addition, in each iteration, we apply structure-oriented regularization to the preconditioned gradients using the structure tensor information Hale (2009); Ma et al. (2010); Williamson et al. (2011). The structure tensors are extracted from the seismic image obtained by ERTM. As shown in Fig. 13, this smoothing provides more balanced and structure-consistent gradients. After 40 iterations for each stage, ERTI provides good recovery of the low-to-intermediate wavenumbers of the $V_{\mathrm{p}}$ and $V_{\mathrm{s}}$ models, see Fig. 14. The results of ERTM are greatly improved by the inverted models and are very close to those obtained with the true models (Fig. 15). In both the PP and $P S$ images, see Figs 15(c) and (d), the diffractions collapse very well, and 


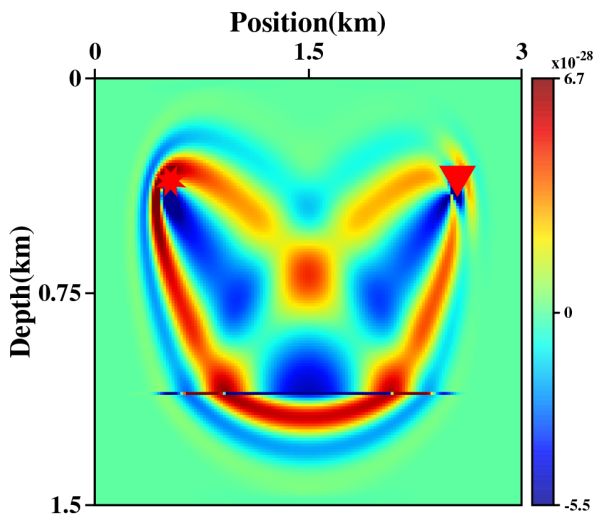

(a)

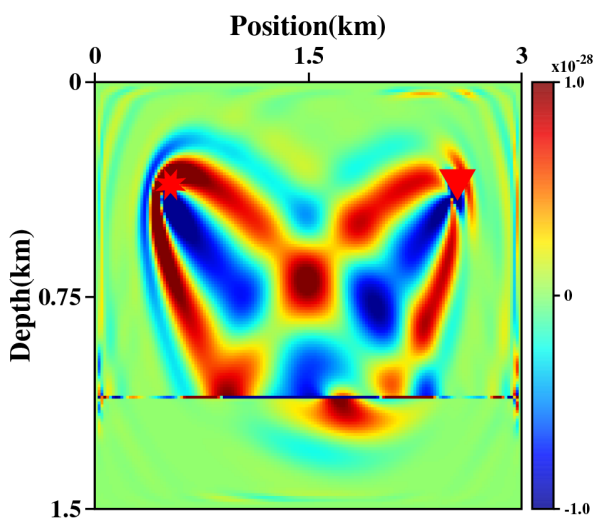

(c)

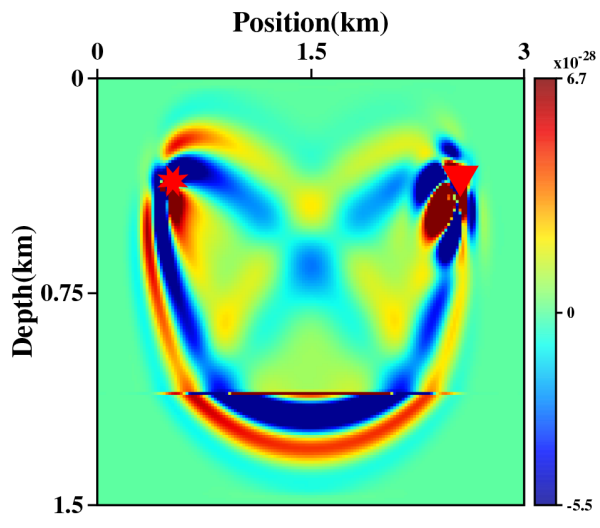

(b)

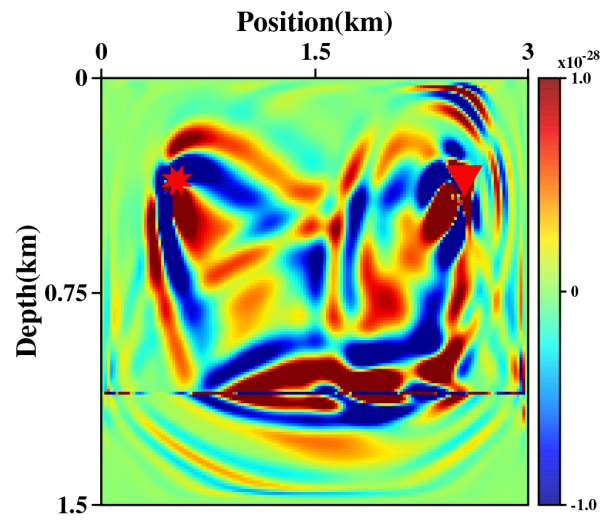

(d)

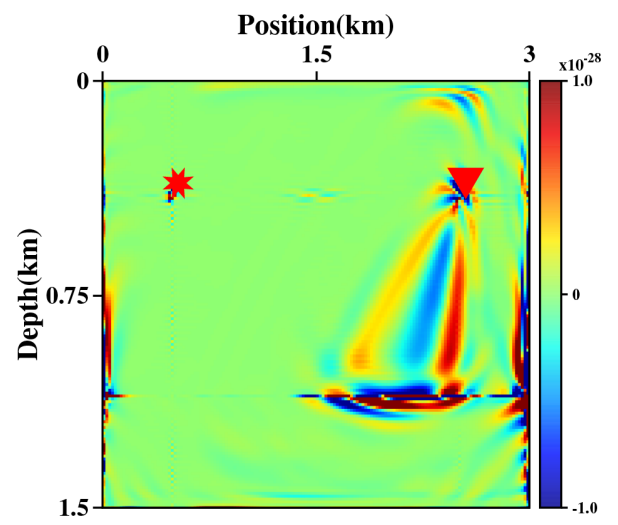

(e)

Figure 21. ERWI kernels using the waveform residual as adjoint source: (a) and (b) are $K_{V_{\mathrm{p}}}$ and $K_{V_{\mathrm{s}}}$ with the settings in Figs 2(c)-(e) are $K_{V_{\mathrm{p}}}, K_{V_{\mathrm{s}}}$ and $K_{V_{\mathrm{s}}}^{S S}$ with the settings in Fig. 3. So we can compare the following pairs to see the differences between the kernel of ERWI and ERTI: (a)-Fig. 2(c), (b)-Fig. 2(d), (c)-Fig. 3(c), (d)-Fig. 3(d) and (e)-Fig. 4(d).

the interfaces including the faults have been migrated to their correct positions. We plot the normalized relative least-squares error of $P P$ and $P S$ traveltime in terms of iterations in Fig. 16. The proposed gradient preconditioning and model regularization methods ensure the convergence of the inversion and help to obtain structureconsistent model updating. Note, in the Stage II, since we used the layer-stripping strategy, the traveltime larger than $2 \mathrm{~s}$ was not used before the 20th iteration. Therefore, the misfit function increases when we included all the data at the 20th iteration.

Fig. 17 shows the $P P$ and $P S$ reflections of observed data and synthetic data through Born modelling with the initial and inverted model. Almost all the PP and $P S$ reflections are well matched with the observed events in kinematics when the inverted models are used. Fig. 18 shows the inverted results of EFWI with the mentioned 


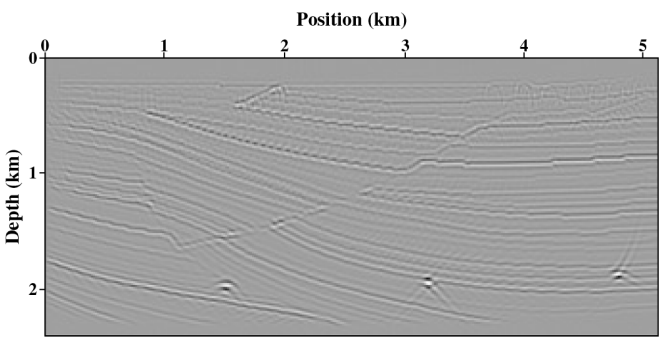

(a)

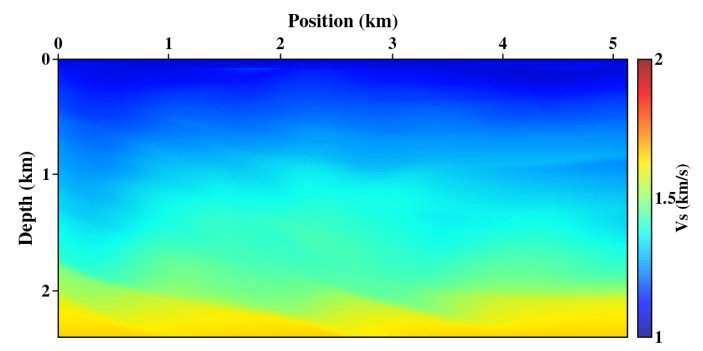

(c)

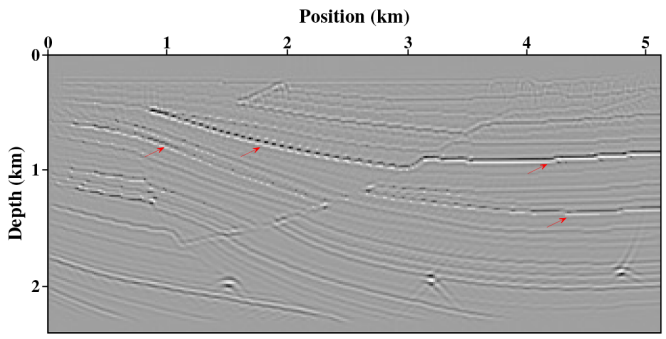

(b)

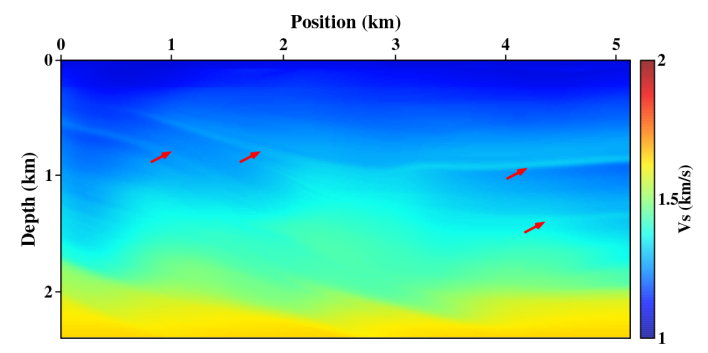

(d)

Figure 22. Inversion test with strong image perturbation: (a) original $\delta V_{\mathrm{s}}$ used in Stage II, (b) $\delta V_{\mathrm{s}}$ with enhancement at certain layers denoted by the red arrows, (c) and (d) $V_{\mathrm{s}}$ model obtained by ERTI with and without model decomposition of extrapolated wavefields. The red arrows in (d) indicate the footprints due to the enhancement in the image perturbation.

hierarchical strategy from low to high frequencies. Compared with Fig. 10, the EFWI with the new starting models shows remarkable improvement, especially in the deeper parts. Therefore, the cycleskipping problem of EFWI is mitigated by initiating the inversion with the velocity models obtained by ERTI. The vertical profiles extracted at 1.4 and $3.0 \mathrm{~km}$ validate the effectiveness of the proposed ERTI approach (Fig. 19). Due to the lack of reflection coverage close to the bottom, we fail to obtain reasonable background models, resulting in poor recovery for this part after EFWI.

Usually, the low-frequency components in the data and the lowto-intermediate wavenumbers in the initial models are key factors in mitigating the cycle-skipping problem in FWI. For field data, the low-frequency components are difficult to obtain and are also more easily contaminated by noise. Therefore, good initial models are important to reduce the nonlinearity caused by the lack of low frequencies. To check the robustness of the above ERTI and EFWI workflow, we test the dependency on the low-frequency data. The low-cut frequency thresholds are 3,5 and $7 \mathrm{~Hz}$. The components lower than the threshold are filtered out for both ERTI and EFWI. As shown in Fig. 20, even when starting from $5 \mathrm{~Hz}$, the final EFWI provides the acceptable inverted results, as in the previous test shown in Fig. 18. Therefore, the low-to-intermediate wavenumber components reconstructed by ERTI help to reduce the dependency of EFWI on low-frequency data.

\section{DISCUSSION}

\subsection{Kernel comparison between ERTI and ERWI}

Although we aim to build the workflow for ERTI, the kernel analyses shown in this paper are also valid for the ERWI workflow. As mentioned at the end of Appendix A, the gradients of ERTI and ERWI share the same calculation form but different in adjoint sources. For brief comparison, we calculate several key (sub)kernels with waveform-type adjoint sources. According to previous analyses, the useful ERTI common-mode kernels mainly concentrate on the first Fresnel zone where the velocity perturbation contributes to the traveltime most. As shown in Fig. 21, the kernels of ERWI show similar appearance but have relatively higher spatial resolution, which means that ERWI can retrieve higher wavenumber components of the model. In the meanwhile, the hollow centre disappears in the common-mode subkernels of $V_{\mathrm{s}}$ using $P$-wave $\left(K_{V_{\mathrm{s}}}^{P P}\right)$. This is reasonable since the waveform information of $P$ wave is more sensitive to the background $V_{\mathrm{s}}$ model. In practice, ERWI would be a complementary stage to the proposed ERTI workflow.

\subsection{Necessity of two-level mode decomposition}

The kernel analyses in Section 3 show that the two-level decomposition removes a lot of artefacts in the gradients. Some of the artefacts can be suppressed by other methods as well, like multishot stacking, smoothing and so on. Here, we try two tests for $S$-wave recovery with the previous Sigsbee2A model to investigate whether the proposed preconditioning methods can be replaced during the inversion. In the first test, we use the data containing both $P$ - and $S$-wave energy to repeat the Stage II inversion. According to eq. (4), DIW measures the traveltime shifts by minimizing the amplitude differences. Generally, the $P P$ reflections are stronger than the $P S$ reflections in seismograms. So, the DIW will focus on matching the $P P$ reflections and thus is unable to find the correct traveltime shifts of $P S$ reflections. Therefore, in this test the gradient with respect to $V_{\mathrm{s}}$ is unacceptable and the step-length estimation always fails. From this perspective, separating the $P P$ and $P S$ reflection events is an essential data preconditioning for traveltime inversion of multicomponent seismic data.

According to Figs 6 and 7, after data preconditioning with $P / S$ separation and dropping the source-side subkernels, the artefacts in $V_{\mathrm{s}}$ kernel have been suppressed greatly. In the second test, we further evaluate the contribution of gradient preconditioning using 
mode decomposition of the extrapolated wavefields. We observe that the subkernels shown in Figs 7(c) and (e) can be suppressed by stacking or smoothing, while the subkernel in Fig. 7(d) cannot. This remaining subkernel will introduce undesirable high-wavenumber components into the background model around the interfaces. These residual artefacts would be more destructive when the reflected $P P$ waves are strong. To verify that, we scale up the image perturbations at certain layers and then repeat the Stage II inversion. In Fig. 22, we observe that the retrieved model contains obvious footprints of the image perturbations if we do not use the second-level mode decomposition. In reality, strong $P P$ reflections due to the velocity contrasts can be easily found in many geological settings. In these situations, it is important to mitigate the high-wavenumber artefacts around the interfaces. Note that mode decomposition of the extrapolated wavefields is a natural way to remove the subkernel in Fig. 7(d), while other methods such as up/down-going wavefield decomposition can-not have a similar effect. If we want to use ERWI for recovering more wavenumber components of $V_{\mathrm{p}}$ and $V_{\mathrm{s}}$ after the proposed ERTI workflow, both $P P$ and $P S$ reflections are required for a simultaneous inversion. In this case, the $P$-wave energy lead to the artefacts in the gradients and parameter trade-off effects if only using the gradient-based methods. So, the second-level mode decomposition would be more useful to precondition the gradient for a better inversion.

\section{CONCLUSION}

In elastic media, background $P$-wave velocities control the traveltime of $P P$ reflections, whereas background $S$-wave velocities determine the receiver-side traveltime of $P S$ reflections. To reconstruct the low-to-intermediate wavenumbers of $P$ - and $S$-wave velocity models, we have extended the approach of wave-equationbased reflection traveltime inversion to elastic media. Based on the adjoint-state method, we derived the gradients with respect to the background velocity models using the traveltime misfit functional with the DIW technique. With the help of a two-level kernel decomposition, we investigate the different features of the complicated reflection kernels in elastic media. Among them, the physical common-mode components present the desirable low wavenumber for ERTI and the other components introduce artefacts. In the physical common-mode components, the $P$-mode components indicate the $P$-wave paths of both $P P$ and $P S$ reflections while the $S$-mode components (mainly in Fig. 7f) indicate the $S$-wave paths of the $P S$ reflections for $V_{\mathrm{s}}$ recovery. To deal with the trade-offs and obtain a desirable update, both the $P / S$ separation of multicomponent seismograms and mode decomposition of the extrapolated wavefields are required.

Based on these observations, we have proposed a gradient-type ERTI approach with a two-stage workflow: the background $P$-wave velocity model is first built using the isolated $P$-wave seismograms, then the background $S$-wave velocity model is built using the isolated $S$-wave seismograms. In the second stage, we introduce mode decomposition to precondition the gradient with respect to the $S$ wave velocity. These data and gradient preconditioning processes provide effective ways to address the nonlinearity of reflection inversion and accelerate the convergence. The Sigsbee2A model example shows that, this wave-equation-based ERTI approach can be used to effectively build migration velocity models for ERTM and to provide appropriate starting models for EFWI.

\section{ACKNOWLEDGEMENTS}

This work is supported by the National Key R\&D Program of China (Grant No. 2017YFB0202903) and National Natural Science Foundation of China (Grant No. 41630964, 41474099 and 41674117). This paper is also based upon the work supported by the King Abdullah University of Science and Technology (KAUST) Office of Sponsored Research (OSR) under award No. 2230. We appreciate the open-source package of DENISE from https://github.com/daniel-koehn/ and Mines Java Toolkit from https://github.com/dhale. We thank the useful advice from Tariq Alkhalifah (KAUST), Zedong Wu (KAUST) and Benxin Chi (Los Alamos). We would like to thank the efforts from Jean Virieux, Wei Zhou and another anonymous reviewer for the revision work of our manuscript.

\section{REFEREN CES}

Almomin, A. \& Biondi, B., 2012. Tomographic full waveform inversion: practical and computationally feasible approach, in SEG Technical Program, Expanded Abstracts, Las Vegas, NV, pp. 1-5.

Biondi, B. \& Almomin, A., 2013. Tomographic full-waveform inversion (TFWI) by combing FWI and wave-equation migration velocity analysis, Leading Edge, 32(9), 1074-1080.

Brossier, R., Operto, S. \& Virieux, J., 2009. Seismic imaging of complex onshore structures by 2D elastic frequency-domain full-waveform inversion, Geophysics, 74(6), WCC105-WCC118.

Broto, K., Ehinger, A., Kommedal, J.H. \& Folstad, P.G., 2003. Anisotropic traveltime tomography for depth consistent imaging of PP and PS data, Leading Edge, 22(2), 114-119.

Chavent, G., Clément, F. \& Gómez, S., 1994. Automatic determination of velocities via migration-based traveltime waveform inversion: a synthetic data example, in SEG Technical Program, Expanded Abstracts, pp. 11791182.

Chi, B., Dong, L. \& Liu, Y., 2015. Correlation-based reflection fullwaveform inversion, Geophysics, 80(4), R189-R202.

Clement, F., Chavent, G. \& Gomez, S., 2001. Migration-based traveltime waveform inversion of 2-D simple structures: a synthetic example, Geophysics, 66, 845-860.

Du, Q., Li, F., Ba, J., Zhu, Y. \& Hou, B., 2012. Multicomponent joint migration velocity analysis in the angle domain for PP-waves and PSwaves, Geophysics, 77(1), U1-U13.

Fichtner, A., Bunge, H.P. \& Igel, H., 2006a. The adjoint method in seismology : I. Theory, Phys. Earth planet. Inter., 157(1-2), 86-104.

Fichtner, A., Bunge, H.P. \& Igel, H., 2006b. The adjoint method in seismology : II. Applications: traveltimes and sensitivity functionals, Phys. Earth planet. Inter., 157(1), 105-123.

Guo, Q. \& Alkhalifah, T., 2017. Elastic reflection based waveform inversion with a nonlinear approach, Geophysics, 82(6), R309-R321.

Guo, Q., Alkhalifah, T. \& Wu, Z., 2017. Velocity building by reflection waveform inversion without cycle-skipping, in 79th EAGE Conference and Exhibition 2017.

Hale, D., 2009. Structure-oriented smoothing and semblance, CWP Rep., 635, Colorado School of Mines, pp. 261-270.

Hale, D., 2013. Dynamic warping of seismic images, Geophysics, 78(2), S105-S115.

Hou, J. \& Symes, W.W., 2015. An approximate inverse to the extended born modeling operator, Geophysics, 80(6), R331-R349.

Jones, I., 2010. Tutorial: velocity estimation via ray-based tomography, First Break, 28(1719), pp. 45-52.

Li, Z., Ma, X., Fu, C., Gu, B. \& Liang, G., 2016. Frequency-wavenumber implementation for P- and S-wave separation from multi-component seismic data, Explor. Geophys., 47(1), 32-43.

Liu, Q. \& Tromp, J., 2006. Finite-frequency kernels based on adjoint methods, Bull. seism. Soc. Am., 96(6), 2383-2397. 
Ma, Y. \& Hale, D., 2013. Wave-equation reflection traveltime inversion with dynamic warping and full waveform inversion, Geophysics, 78(6), R223-R233.

Ma, Y., Hale, D., Meng, Z. \& Gong, B., 2010. Full waveform inversion with image-guided gradient, in SEG Technical Program, Expanded Abstracts, Denver, CO, pp. 1003-1007.

Oh, J.-W. \& Alkhalifah, T., 2016. The scattering potential of partial derivative wavefields in 3-D elastic orthorhombic media: an inversion prospective, Geophys. J. Int., 206(3), 1740-1760.

Operto, S., Gholami, Y., Prieux, V., Ribodetti, A., Brossier, R., Metivier, L. \& Virieux, J., 2013. A guided tour of multiparameter full-waveform inversion with multicomponent data: from theory to practice, Leading Edge, 32(9), 1040-1054.

Plessix, R.-E., 2006. A review of the adjoint-state method for computing the gradient of a functional with geophysical applications, Geophys. J. Int., 167(2), 495-503.

Plessix, R.-E., Roeck, Y.-H.D. \& Chavent, G., 1999. Waveform inversion of reflection seismic data for kinematic parameters by local optimization, SIAM J. Sci. Comput., 20(3), 1033-1052.

Pratt, R.G., Shin, C. \& Hick, G., 1998. Gauss-Newton and full Newton methods in frequency-space seismic waveform inversion, Geophys. $J$. Int., 133(2), 341-362.

Prieux, V., Brossier, R., Operto, S. \& Virieux, J., 2013. Multiparameter full waveform inversion of multicomponent ocean-bottom-cable data from the valhall field. Part 2: imaging compressive-wave and shear-wave velocities, Geophys. J. Int., 194(3), 1665-1681.

Raknes, E.B. \& Weibull, W., 2016. Combining wave-equation migration velocity analysis and full-waveform inversion for improved 3D elastic parameter estimation, in SEG International Exposition and Annual Meeting, Dallas, TX.

Sears, T., Singh, S. \& Barton, P., 2008. Elastic full waveform inversion of multi-component OBC seismic data, Geophys. Prospect., 56, 843-862.

Shipp, R.M. \& Singh, S.C., 2002. Two-dimensional full wavefield inversion of wide-aperture marine seismic streamer data, Geophys. J. Int., 151(2), 325-344.

Stork, C., 1992. Reflection tomography in the postmigrated domain, Geophysics, 57(5), 680-692.

Sun, D. \& Symes, W.W., 2012. Waveform inversion via nonlinear differential semblance optimization, in SEG Annual Meeting, Expanded Abstracts, Las Vegas, NV, pp. 1-7.

Symes, W.W., 2008a. Approximate linearized inversion by optimal scaling of prestack depth migration, Geophysics, 73(2), R23-R35.

Symes, W.W., 2008b. Migration velocity analysis and waveform inversion, Geophys. Prospect., 56(6), 765-790.

Tarantola, A., 1984. Inversion of seismic reflection data in the acoustic approximation, Geophysics, 49(8), 1259-1266.

Tarantola, A., 1986. A strategy for nonlinear elastic inversion of seismic reflection data, Geophysics, 51(10), 1893-1903.

Tromp, J., Tape, C. \& Liu, Q., 2005. Seismic tomography, adjoint methods, time reversal and banana-doughnut kernels, Geophys. J. R. astr. Soc., 160(1), 195-216.

Virieux, J. \& Operto, S., 2009. An overview of full-waveform inversion in exploration geophysics, Geophysics, 74(6), WCC1-WCC26.

Wang, T. \& Cheng, J., 2017. Elastic full waveform inversion based on mode decomposition: the approach and mechanism, Geophys. J. Int., 209(2), 606-622.

Wang, T., Kang, W. \& Cheng, J., 2014. Migration velocity model building using local angle domain nonlinear tomography, in Beijing 2014 International Geophysical Conference \& Exposition, Beijing, China, pp. 739-742.

Wang, H., Singh, S.C., Audebert, F. \& Calandra, H., 2015a. Inversion of seismic refraction and reflection data for building long-wavelength velocity models, Geophysics, 80(2), R81-R93.
Wang, T., Cheng, J. \& Wang, C., 2015b. Elastic wave mode decoupling for full waveform inversion, in 77th EAGE Conference and Exhibition 2015, Expanded Abstracts, New Orleans, LA, pp. 1461-1466.

Wang, C., Weibull, W., Cheng, J. \& Arntsen, B., 2017a. Automatic shearwave velocity analysis with elastic reverse time migration, in 79th EAGE Conference and Exhibition 2017, Expanded Abstracts.

Wang, T., Cheng, J., Guo, Q. \& Wang, C., 2017b. Elastic wave-equation reflection traveltime inversion using dynamic warping and wave mode decomposition, in 79th EAGE Conference and Exhibition 2017, Expanded Abstracts.

Weibull, W.W. \& Arntsen, B., 2014. Reverse-time demigration using the extended-imaging condition, Geophysics, 79(3), WA97-WA105.

Williamson, P., Atle, A., Fei, W. \& Hale, D., 2011. Regularization of waveequation migration velocity analysis by structure-oriented smoothing, in SEG Annual Meeting, Expanded Abstracts, San Antonio, TX, pp. $3877-$ 3881.

Woodward, M.J., Nichols, D., Zdraveva, O., Whitfield, P. \& Johns, T., 2008. A decade of tomography, Geophysics, 73(5), VE5-VE11.

Wu, Z. \& Alkhalifah, T., 2015. Simultaneous inversion of the background velocity and the perturbation in full-waveform inversion, Geophysics, 80(6), R317-R329.

Xu, S., Wang, D., Chen, F., Lambare, G. \& Zhang, Y., 2012. Inversion on reflected seismic wave, in 82nd Annual International Meeting, SEG, Expanded Abstracts, pp. 1-7.

Yang, D., Shang, X., Malcolm, A., Fehler, M. \& Baek, H., 2015. Image registration guided wavefield tomography for shear-wave velocity model building, Geophysics, 80(3), U35-U46.

Zhou, W., Brossier, R., Operto, S. \& Virieux, J., 2015. Full waveform inversion of diving \& reflected waves for velocity model building with impedance inversion based on scale separation, Geophys. J. Int., 202, $1535-1554$

\section{APPENDIX A: ADJOINT-STATE METHOD FOR ERTI}

In this appendix, we will derive the gradients of ERTI using adjointstate method Liu \& Tromp (2006); Plessix (2006); Ma \& Hale (2013). According to Hale (2013), the $L 2$ distance between observed and calculated data satisfies

$D(\tau)=\frac{1}{2} \int_{0}^{T} \sum_{r}\left[\mathbf{d}^{c}\left(\mathbf{x}_{r}, t\right)-\mathbf{d}^{o}\left(\mathbf{x}_{r}, t+\tau\left(\mathbf{x}_{r}, t\right)\right)\right]^{2} \mathrm{~d} t$.

DIW aims to find $\tau \equiv \xi\left(\mathbf{x}_{r}, t\right)$ to minimize the objective function $D(\xi)$, which implies

$\frac{\partial D}{\partial \tau}=\int_{0}^{T} \sum_{r} \alpha\left(\mathbf{x}_{r}, t\right) \mathrm{d} t=0$,

where $\alpha\left(\mathbf{x}_{r}, t\right)=\dot{\mathbf{d}}^{o}\left(\mathbf{x}_{r}, t+\tau\right)\left(\mathbf{d}^{o}\left(\mathbf{x}_{r}, t+\tau\right)-\mathbf{d}^{c}\left(\mathbf{x}_{r}, t\right)\right.$. For simplicity in the derivation, we rewrite eqs (2) and (3) as

$\rho \partial_{t}^{2} \mathbf{u}-\nabla \cdot\left(\mathbf{c}^{0}: \nabla \mathbf{u}\right)=\mathbf{f}$ and

$\rho \partial_{t}^{2} \hat{\mathbf{u}}-\nabla \cdot\left(\mathbf{c}^{0}: \nabla \hat{\mathbf{u}}\right)=\nabla \cdot\left(\mathbf{c}^{1}: \nabla \mathbf{u}\right)$,

where $\partial_{t}^{2}$ is the second-order time derivative. Eqs (A2) and (A3) are the state equations in ERTI. Our objective is to minimize the misfit function, eq. (4), subject to the constraints that the background and perturbed wavefields ( $\mathbf{u}$ and $\hat{\mathbf{u}}$ ) satisfy the wave eq. (A3), and the time-shift $\tau$ satisfies eq. (A2). Therefore, using eqs (A3) and (A2) 
we can define the Lagrangian $\mathcal{L}$ :

$$
\begin{aligned}
\mathcal{L} & =\frac{1}{2} \sum_{r} \int_{0}^{T} \tau^{2}\left(\mathbf{x}_{r}, t\right) \mathrm{d} t-\sum_{r} \int_{0}^{T} \mu\left(\mathbf{x}_{r}, t\right) \alpha\left(\mathbf{x}_{r}, t\right) \mathrm{d} t \\
& -\int_{0}^{T} \int_{\Omega} \hat{\psi}\left[\rho \partial_{t}^{2} \mathbf{u}-\nabla \cdot\left(\mathbf{c}^{0}: \nabla \mathbf{u}\right)-\mathbf{f}\right] \mathrm{d}^{3} \mathbf{x} \mathrm{d} t \\
& -\int_{0}^{T} \int_{\Omega} \psi\left[\rho \partial_{t}^{2} \hat{\mathbf{u}}-\nabla \cdot\left(\mathbf{c}^{0}: \nabla \hat{\mathbf{u}}\right)-\nabla \cdot\left(\mathbf{c}^{1}: \nabla \mathbf{u}\right)\right] \mathrm{d}^{3} \mathbf{x} \mathrm{d} t,
\end{aligned}
$$

in which $\Omega$ is the integration domain, $\tau$, $\mathbf{u}$ and $\hat{\mathbf{u}}$ are state variables, and $\mu, \psi$ and $\hat{\psi}$ are Lagrange multipliers (or adjoint-state variables) that remain to be determined. Note that the predicted reflection data are the forward-perturbed wavefields at the receivers, that is, $\mathbf{d}^{c}\left(\mathbf{x}_{r}, t\right)=\delta\left(\mathbf{x}-\mathbf{x}_{r}\right) \delta \hat{\mathbf{u}}$. Taking the variation of the action (A4), we obtain

$$
\begin{aligned}
\delta \mathcal{L} & =\int_{0}^{T} \sum_{r}\left[\tau\left(\mathbf{x}_{r}, t\right)-\mu\left(\mathbf{x}_{r}, t\right) h\left(\mathbf{x}_{r}, t\right)\right] \cdot \delta \boldsymbol{\tau} \mathrm{d} t \\
& +\int_{0}^{T} \int_{\Omega} \mu\left(\mathbf{x}_{r}, t\right) \dot{\mathbf{d}}^{o}\left(\mathbf{x}_{r}, t+\boldsymbol{\tau}\right) \delta\left(\mathbf{x}-\mathbf{x}_{r}\right) \cdot \delta \hat{\mathbf{u}} d^{3} \mathbf{x} \mathrm{d} t \\
& +\int_{0}^{T} \int_{\Omega}\left[\hat{\psi} \nabla \cdot\left(\delta \mathbf{c}^{0}: \nabla \mathbf{u}\right)+\boldsymbol{\psi} \nabla \cdot\left(\delta \mathbf{c}^{0}: \nabla \hat{\mathbf{u}}\right)\right] \mathrm{d}^{3} \mathbf{x} \mathrm{d} t \\
& -\int_{0}^{T} \int_{\Omega} \hat{\psi}\left[\rho \partial_{t}^{2} \delta \mathbf{u}-\nabla \cdot\left(\mathbf{c}^{0}: \nabla \delta \mathbf{u}\right)\right] \mathrm{d}^{3} \mathbf{x} \mathrm{d} t \\
& -\int_{0}^{T} \int_{\Omega} \boldsymbol{\psi}\left[\rho \partial_{t}^{2} \delta \hat{\mathbf{u}}-\nabla \cdot\left(\mathbf{c}^{0}: \nabla \delta \hat{\mathbf{u}}\right)-\nabla \cdot\left(\mathbf{c}^{1}: \nabla \delta \mathbf{u}\right)\right] \mathrm{d}^{3} \mathbf{x} \mathrm{d} t,
\end{aligned}
$$

where $\quad h\left(\mathbf{x}_{\mathbf{r}}, t\right)=\dot{\mathbf{d}}^{o}\left(\mathbf{x}_{\mathbf{r}}, t+\tau\right)^{2}-\ddot{\mathbf{d}}^{o}\left(\mathbf{x}_{\mathbf{r}}, t+\tau\right)\left[\mathbf{d}^{c}\left(\mathbf{x}_{\mathbf{r}}, t\right)-\right.$ $\left.\mathbf{d}^{o}\left(\mathbf{x}_{\mathbf{r}}, t+\tau\right)\right]$.

Upon integrating the terms involving spatial and temporal derivatives of $\mathbf{u}, \delta \mathbf{u}$, $\mathbf{u}$ and $\delta \hat{\mathbf{u}}$ by parts, we have

$$
\begin{aligned}
& \delta \mathcal{L}=\int_{0}^{T} \sum_{r}\left[\tau\left(\mathbf{x}_{r}, t\right)-\mu\left(\mathbf{x}_{r}, t\right) h\left(\mathbf{x}_{r}, t\right)\right] \cdot \delta \tau \mathrm{d} t \\
& +\int_{0}^{T} \int_{\Omega} \mu\left(\mathbf{x}_{r}, t\right) \dot{\mathbf{d}}^{o}\left(\mathbf{x}_{r}, t+\boldsymbol{\tau}\right) \delta\left(\mathbf{x}-\mathbf{x}_{r}\right) \cdot \delta \hat{\mathbf{u}} \mathrm{d}^{3} \mathbf{x} \mathrm{d} t \\
& -\int_{0}^{T} \int_{\Omega}\left[\nabla \hat{\psi}: \delta \mathbf{c}^{0}: \nabla \mathbf{u}+\nabla \psi: \delta \mathbf{c}^{0}: \nabla \hat{\mathbf{u}}\right] \mathrm{d}^{3} \mathbf{x} \mathrm{d} t \\
& -\int_{0}^{T} \int_{\Omega}\left[\rho \partial_{t}^{2} \hat{\boldsymbol{\psi}}-\nabla \cdot\left(\mathbf{c}^{0}: \nabla \hat{\boldsymbol{\psi}}\right)-\nabla \cdot\left(\mathbf{c}^{1}: \nabla \boldsymbol{\psi}\right)\right] \delta \mathbf{u d}^{3} \mathbf{x} \mathrm{d} t \\
& -\int_{0}^{T} \int_{\Omega}\left[\rho \partial_{t}^{2} \boldsymbol{\psi}-\nabla \cdot\left(\mathbf{c}^{0}: \nabla \boldsymbol{\psi}\right)\right] \delta \hat{\mathbf{u}} \mathrm{d}^{3} \mathbf{x} \mathrm{d} t \\
& -\int_{\Omega}\left[\rho\left(\hat{\boldsymbol{\psi}} \cdot \partial_{t} \delta \mathbf{u}-\partial_{t} \hat{\boldsymbol{\psi}} \cdot \delta \mathbf{u}+\boldsymbol{\psi} \cdot \partial_{t} \delta \hat{\mathbf{u}}-\partial_{t} \boldsymbol{\psi} \cdot \delta \hat{\mathbf{u}}\right)\right]_{0}^{T} \mathrm{~d}^{3} \mathbf{x} \\
& +\int_{0}^{T} \int_{\partial \Omega} \hat{\psi} \cdot\left[\mathbf{n} \cdot\left(\delta \mathbf{c}^{0}: \nabla \mathbf{u}+\mathbf{c}^{0}: \nabla \delta \mathbf{u}\right)\right] \\
& -\mathbf{n} \cdot\left(\mathbf{c}^{0}: \nabla \hat{\psi}\right) \cdot \delta \mathbf{u} \mathrm{d}^{2} \mathbf{x} \mathrm{d} t \\
& +\int_{0}^{T} \int_{\partial \Omega} \boldsymbol{\psi} \cdot\left[\mathbf{n} \cdot\left(\delta \mathbf{c}^{0}: \nabla \hat{\mathbf{u}}+\mathbf{c}^{0}: \nabla \delta \hat{\mathbf{u}}\right)\right] \\
& -\mathbf{n} \cdot\left(\mathbf{c}^{0}: \nabla \boldsymbol{\psi}\right) \cdot \delta \hat{\mathbf{u}} \mathrm{d}^{2} \mathbf{x} \mathrm{d} t \\
& +\int_{0}^{T} \int_{\partial \Omega} \boldsymbol{\psi} \cdot\left[\mathbf{n} \cdot\left(\mathbf{c}^{1}: \nabla \delta \mathbf{u}\right)\right]-\mathbf{n} \cdot\left(\mathbf{c}^{1}: \nabla \boldsymbol{\psi}\right) \cdot \delta \mathbf{u d}^{2} \mathbf{x} \mathrm{d} t
\end{aligned}
$$

where $\mathbf{n}$ is the unit outward vector normal on the surface $\partial \Omega$. The regular wavefields are subject to the initial and boundary condition:

$$
\begin{aligned}
& \mathbf{u}(\mathbf{x}, 0)=0, \partial_{t} \mathbf{u}(\mathbf{x}, 0)=0,\left.\mathbf{u}(\mathbf{x}, t)\right|_{x \rightarrow \infty} \rightarrow 0 \\
& \hat{\mathbf{u}}(\mathbf{x}, 0)=0, \partial_{t} \hat{\mathbf{u}}(\mathbf{x}, 0)=0,\left.\hat{\mathbf{u}}(\mathbf{x}, t)\right|_{x \rightarrow \infty} \rightarrow 0,
\end{aligned}
$$

while the adjoint wavefields satisfy the 'final' (at the time of $T$ ) and boundary condition:

$$
\begin{aligned}
& \boldsymbol{\psi}(\mathbf{x}, T)=0, \partial_{t} \boldsymbol{\psi}(\mathbf{x}, T)=0,\left.\boldsymbol{\psi}(\mathbf{x}, t)\right|_{x \rightarrow \infty} \rightarrow 0 \text { and } \\
& \hat{\psi}(\mathbf{x}, T)=0, \partial_{t} \hat{\boldsymbol{\psi}}(\mathbf{x}, T)=0,\left.\hat{\boldsymbol{\psi}}(\mathbf{x}, t)\right|_{x \rightarrow \infty} \rightarrow 0,
\end{aligned}
$$

on $\partial \Omega$. Thus all the surface integrals in eq. (A6) will disappear, then we have

$$
\begin{aligned}
\delta \mathcal{L} & =\int_{0}^{T} \sum_{r}\left[\tau\left(\mathbf{x}_{r}, t\right)-\mu\left(\mathbf{x}_{r}, t\right) h\left(\mathbf{x}_{r}, t\right)\right] \delta \tau \mathrm{d} t \\
& -\int_{0}^{T} \int_{\Omega}\left[\rho \partial_{t}^{2} \hat{\boldsymbol{\psi}}-\nabla \cdot\left(\mathbf{c}^{0}: \nabla \hat{\boldsymbol{\psi}}\right)-\nabla \cdot\left(\mathbf{c}^{1}: \nabla \boldsymbol{\psi}\right)\right] \delta \mathbf{u} \mathrm{d}^{3} \mathbf{x} \mathrm{d} t \\
& -\int_{0}^{T} \int_{\Omega}\left[\rho \partial_{t}^{2} \boldsymbol{\psi}-\nabla \cdot\left(\mathbf{c}^{0}: \nabla \boldsymbol{\psi}\right)\right. \\
& \left.-\mu\left(\mathbf{x}_{r}, t\right) \dot{\mathbf{d}}^{o}\left(\mathbf{x}_{r}, t+\tau\right) \delta\left(\mathbf{x}-\mathbf{x}_{r}\right)\right] \delta \hat{\mathbf{u}}^{3} \mathbf{x} \mathrm{d} t \\
& -\int_{0}^{T} \int_{\Omega}\left[\left(\nabla \hat{\boldsymbol{\psi}}: \delta \mathbf{c}^{0}: \nabla \mathbf{u}\right)+\left(\nabla \boldsymbol{\psi}: \delta \mathbf{c}^{0}: \nabla \hat{\mathbf{u}}\right)\right] \mathrm{d}^{3} \mathbf{x} \mathrm{d} t .
\end{aligned}
$$

To obtain the stationary points, setting the coefficients of $\delta \tau$ to zero yields

$\mu\left(\mathbf{x}_{r}, t\right)=\frac{\tau\left(\mathbf{x}_{r}, t\right)}{h\left(\mathbf{x}_{r}, t\right)}$,

and setting $\delta \mathbf{u}$ and $\delta \hat{\mathbf{u}}$ to zero yields another two adjoint state equations:

$$
\begin{aligned}
\rho \partial_{t}^{2} \boldsymbol{\psi}-\nabla \cdot\left(\mathbf{c}^{0}: \nabla \boldsymbol{\psi}\right) & =\mu\left(\mathbf{x}_{r}, t\right) \dot{\mathbf{d}}^{o}\left(\mathbf{x}_{r}, t+\tau\right) \delta\left(\mathbf{x}-\mathbf{x}_{r}\right) \text { and } \\
\rho \partial_{t}^{2} \hat{\boldsymbol{\psi}}-\nabla \cdot\left(\mathbf{c}^{0}: \nabla \hat{\boldsymbol{\psi}}\right) & =\nabla \cdot\left(\mathbf{c}^{1}: \nabla \boldsymbol{\psi}\right) .
\end{aligned}
$$

The first equation implies that the adjoint background wavefields $\psi$ is determined by the adjoint source at the receiver locations; while the second equation implies that the adjoint perturbed wavefields $\hat{\psi}$ is determined by the virtual source inspired by the high-wavenumber image perturbations. The adjoint wavefields $\psi$ and $\hat{\psi}$ can be obtained by solving eq. (A11) in a time-reversed manner. Therefore, eq. (A9) implies that we have the gradient

$\frac{\partial \mathcal{L}}{\partial \mathbf{c}^{0}}=-\int_{0}^{T} \int_{\Omega}[\nabla \hat{\boldsymbol{\psi}} \nabla \mathbf{u}+\nabla \boldsymbol{\psi} \nabla \hat{\mathbf{u}}] \mathrm{d}^{3} \mathbf{x} \mathrm{d} t$,

or in a more detailed manner:

$\frac{\partial E}{\partial c_{i j k l}^{0}}=-\int\left(\frac{\partial u_{i}}{\partial x_{j}} \frac{\partial \hat{\psi}_{k}}{\partial x_{l}}+\frac{\partial \hat{u}_{i}}{\partial x_{j}} \frac{\partial \psi_{k}}{\partial x_{l}}\right) \mathrm{d} t$.

In fact, the gradient in ERWI shares the same forms as in eqs (A12) and (A13), so long as the adjoint background wavefields backward propagate from the adjoint sources directly induced by the waveform residuals at the receiver locations, namely $\mathbf{d}^{c}\left(\mathbf{x}_{\mathbf{r}}, t\right)-\mathbf{d}^{o}\left(\mathbf{x}_{\mathbf{r}}, t\right)$.

\section{APPENDIX B: SECOND-LEVEL DECOMPOSITION OF THE OTHER THREE SUBKERNELS OF $V$ s}

For integrity, we illustrate the second-level decomposition of the other three subkernels for $K_{V_{\mathrm{s}}}$ in Fig. 6. Initially, we investigate the 
two source-side subkernels in Figs 6(e) and (f). In fact, the nonphysical mode conversions at the receiver locations introduce both $P$ and $S$ wavefields into the adjoint background wavefield $(\psi)$. That will make the adjoint perturbed wavefields $(\hat{\psi})$ extremely complex after they are reflected and converted at the interface. To simplify the classification of these source-side subkernels, we do not distinguish the wave mode of $\boldsymbol{\psi}$ but only distinguish the wave mode of $\hat{\psi}$ before the cross-correlation. As shown in Fig. B1, the source-side subkernels are decomposed into two components for injection of $P P$ and $P S$ events. In this case, cross-mode cross-correlations induce artefacts in the reflection kernel (Figs B1 f and h), while commonmode cross-correlations mainly generate low-wavenumber components along the source-side wave paths (Figs B1 e and g). Because the $S$ waves in the adjoint perturbed wavefields stem from mode conversions both at the interface and on the recording surface (when injecting the adjoint sources), the cross-mode subkernels possess both low and relatively high wavenumbers. Decomposing them by distinguishing their origins is possible but requires greater computational efforts. For injection of the isolated $P P$ event, the source-side kernels of $V_{\mathrm{p}}$ (Fig. 5e) and $V_{\mathrm{s}}$ (Fig. B1e) have similar shapes but opposite polarities in the first Fresnel zone, except that the latter has a hollow centre. Additionally, for injection of the isolated PS event, the source-side kernels of $V_{\mathrm{p}}$ (Fig. 5f) and $V_{\mathrm{s}}$ (Fig. B1g) have similar shapes but opposite polarities in the first Fresnel zone because the terms (with $\delta_{i j} \delta_{k l}$ ) of divergence operation in the gradient calculation have opposite signs, see eq. (9).

Then we apply the second-level decomposition to the subkernel in Fig. 6(g). The injected PP event generates non-physical PS waves from the receiver locations. Similarly, non-physical mode conversion-related common-mode cross-correlations and crossmode cross-correlations induce artefacts in the reflection kernel (Figs B2 d-f). The receiver-side kernels of $V_{\mathrm{p}}$ (Fig. $5 \mathrm{~g}$ ) and $V_{\mathrm{s}}$ (Fig. B2c) also share the same similarities and differences as that in the source-side ones due to the previously mentioned reason.

(a)

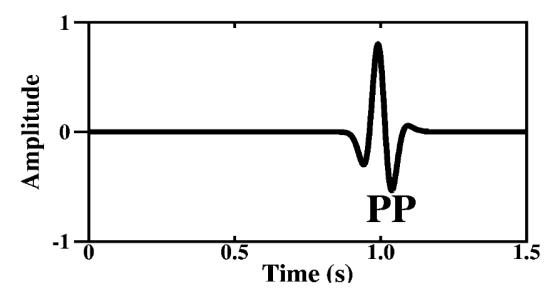

(b)
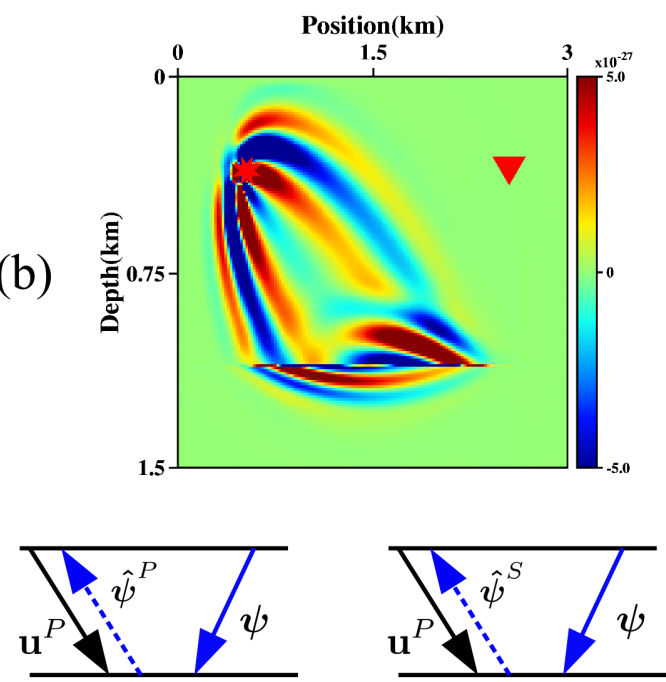

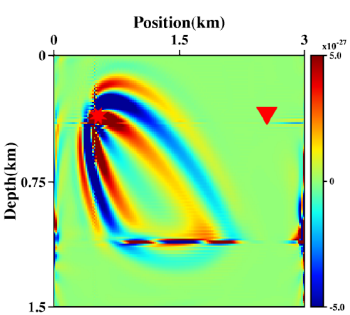

(e)

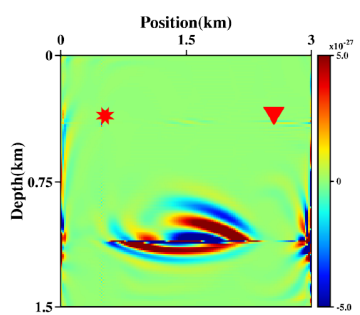

(f)

(c)

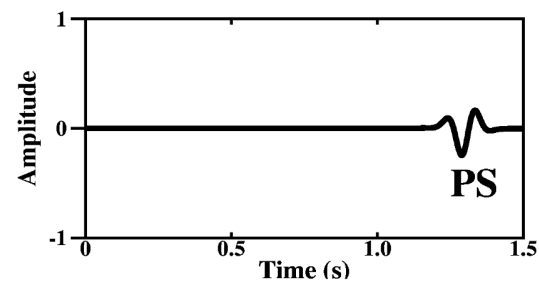

(d)
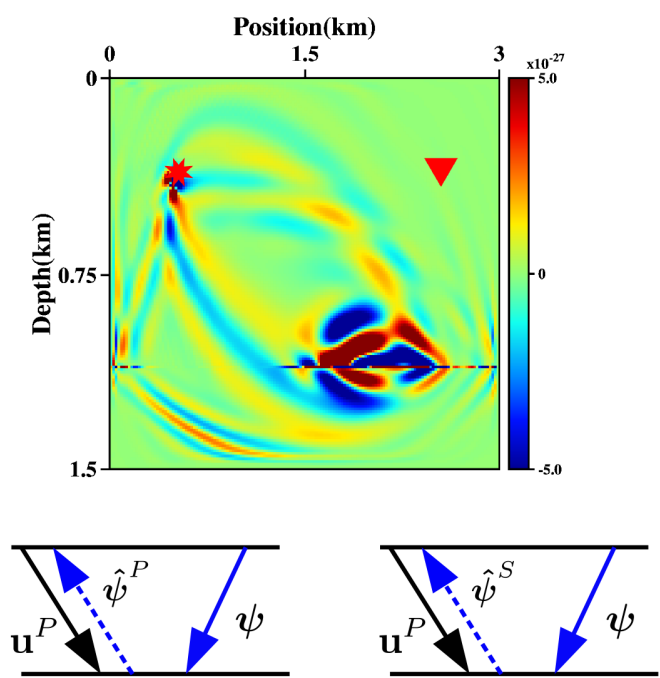

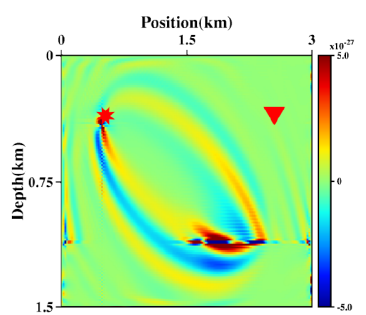

(g)

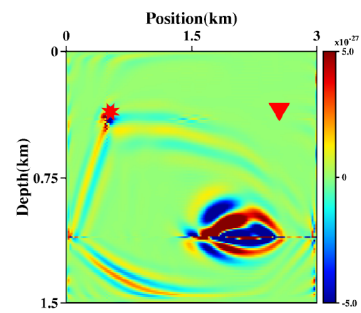

(h)

Figure B1. Further decomposition of the two source-side subkernels in Figs 6(e) and (f): panels (a) and (c) denote the isolated adjoint sources; panels (b) and (d) represent the source-side subkernels to be decomposed; panels (e)-(h) show the involved wave modes and the corresponding kernel components. 
(a)

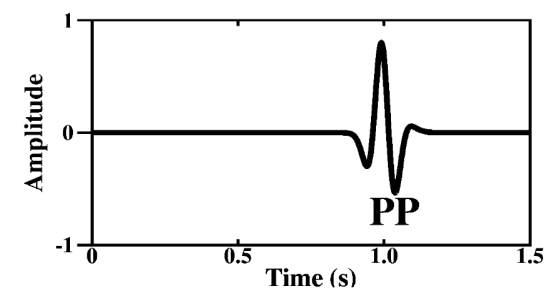

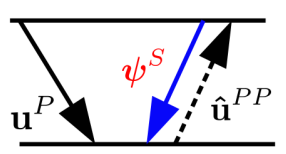

(b)
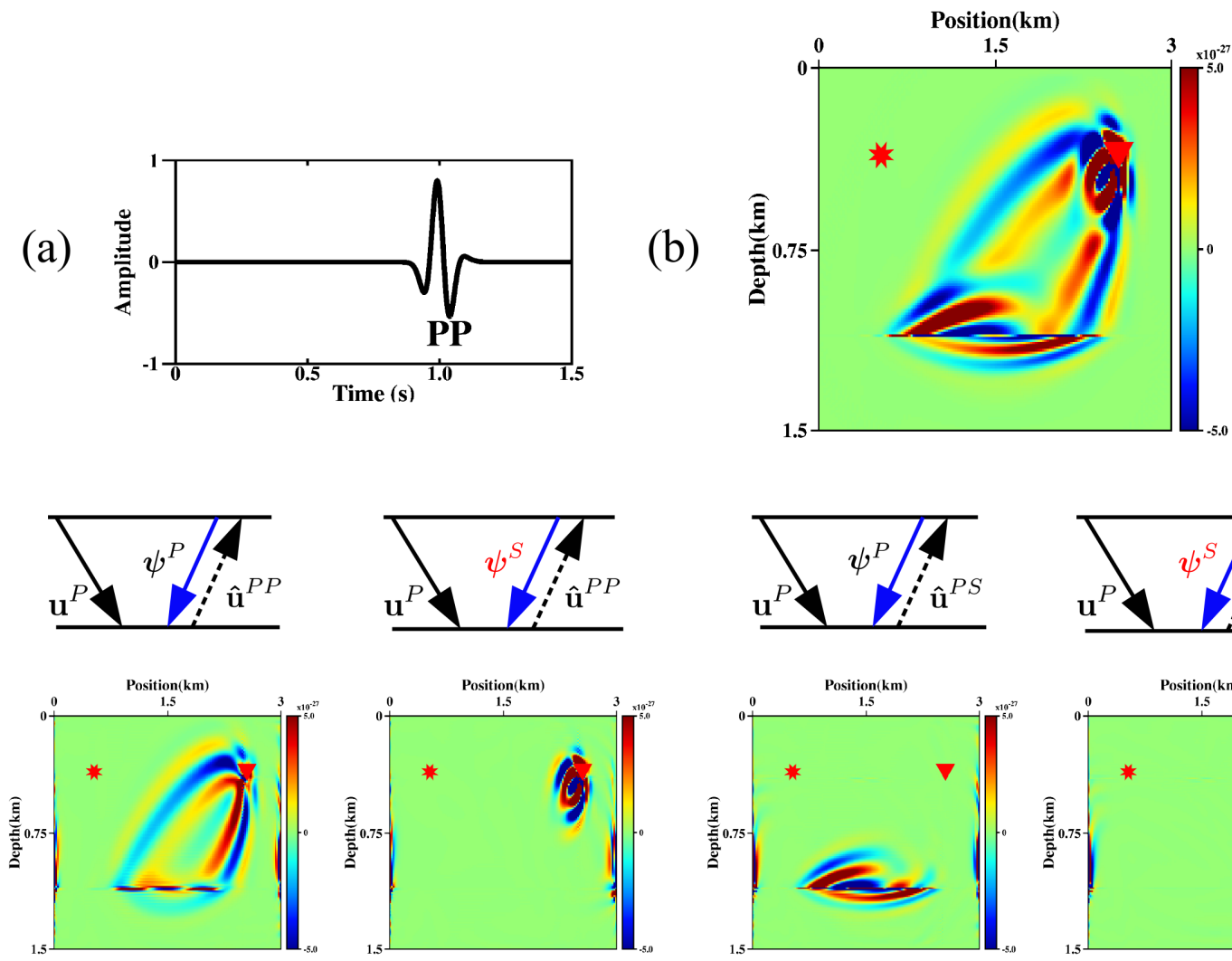

(c)

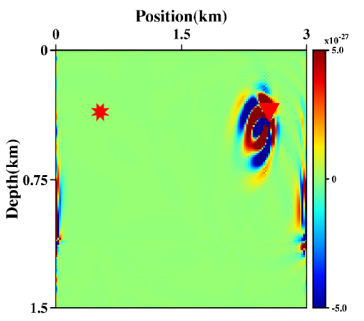

(d)
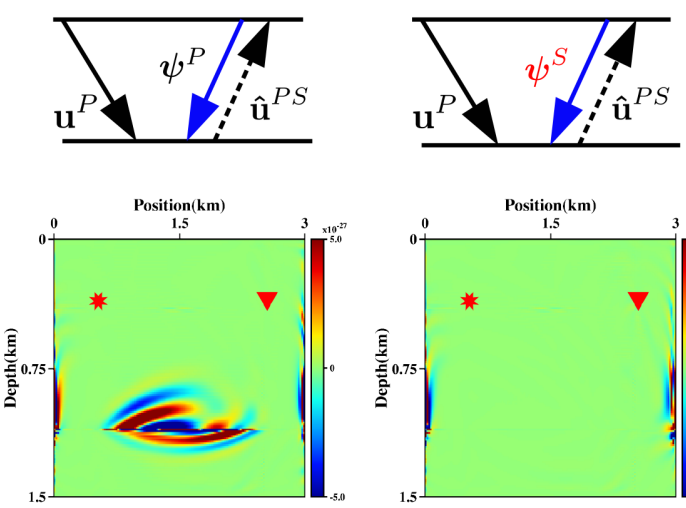

(e)

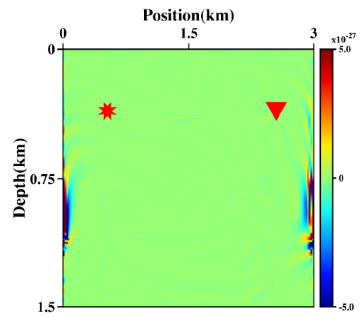

(f)

Figure B2. Further decomposition of the remaining receiver-side subkernel in Fig. 6(g): panel (a) denotes the isolated adjoint source; panel (b) represents the subkernel to be decomposed; panels (c)-(f) show the involved wave modes and the corresponding kernel components. Note that the red symbols $\left(\psi^{S}\right)$ in $(\mathrm{d})$ and (f) denote the backward propagating $P S$ waves resulting from the non-physical mode conversion at the receiver location. 